\title{
Inflationary non-Gaussianities in the most general second-order scalar-tensor theories
}

\author{
Antonio De Felice and Shinji Tsujikawa \\ Department of Physics, Faculty of Science, Tokyo University of Science, \\ 1-3, Kagurazaka, Shinjuku-ku, Tokyo 162-8601, Japan
}

(Dated: October 30, 2018)

\begin{abstract}
For very general scalar-field theories in which the equations of motion are at second-order, we evaluate the three-point correlation function of primordial scalar perturbations generated during inflation. We show that the shape of non-Gaussianities is well approximated by the equilateral type. The equilateral non-linear parameter $f_{\mathrm{NL}}^{\text {equil }}$ is derived on the quasi de Sitter background where the slow-variation parameters are much smaller than unity. We apply our formula for $f_{\mathrm{NL}}^{\text {equil }}$ to a number of single-field models of inflation-such as k-inflation, k-inflation with Galileon terms, potential-driven Galileon inflation, nonminimal coupling models (including field-derivative coupling models), and Gauss-Bonnet gravity.
\end{abstract}

\section{INTRODUCTION}

The inflationary paradigm [1] can successfully account for the observed temperature anisotropies in Cosmic Microwave Background (CMB) [2] as well as the galaxy power spectrum [3]. Although we have not identified the origin of inflation yet, the upcoming observations such as PLANCK [4] are expected to provide more high-precision data for the primordial scalar/tensor power spectra. This will allow us to distinguish between a host of theoretical models of inflation [5] observationally.

In addition to the spectral index of scalar density perturbations $n_{\mathcal{R}}$ and the tensor-to-scalar ratio $r$, the information of primordial scalar non-Gaussianities is useful to break the degeneracy between different models [6-9]. In standard slow-roll inflation in which cosmic acceleration is driven by the potential energy of a scalar field $\phi$, the non-linear parameter $f_{\mathrm{NL}}^{\text {equil }}$ characterizing the equilateral shape of non-Gaussianities is small $\left(\left|f_{\mathrm{NL}}^{\text {equil }}\right| \ll 1\right)$ [10, 11] (see also Refs. $12-14 \mid)$. Meanwhile, in the presence of a non-linear field kinetic energy in $X=-\partial^{\mu} \phi \partial_{\mu} \phi / 2$ such as k-inflation [15], Dirac-Born-Infeld (DBI) models [16, 17], (dilatonic) ghost condensate [18, 19], and Galileon inflation [20, 21], it is possible to realize large non-Gaussianities detectable in future observations $\left(\left|f_{\mathrm{NL}}^{\text {equil }}\right| \gtrsim 10\right)[22[25]$.

The viable theoretical models of inflation are usually constructed to keep the equations of motion at second-order in order to avoid the Ostrogradski's instability [26]. In 1974 Horndeski 27] derived the most general single-field Lagrangian giving rise to second-order field equations. Recently there has been renewed interest in second-order gravitational theories in connection with the Dvali-Gabadadze-Porrati braneworld [28] and Galileon gravity [29, 30] (see also Refs. [31-40]). Deffayet et al. [41] showed that the most general action in those theories is given by

$$
S=\int d^{4} x \sqrt{-g}\left[\frac{M_{\mathrm{pl}}^{2}}{2} R+P(\phi, X)-G_{3}(\phi, X) \square \phi+\mathcal{L}_{4}+\mathcal{L}_{5}\right] .
$$

Here $g$ is the determinant of the metric $g_{\mu \nu}, M_{\mathrm{pl}}$ is the reduced Planck mass, $R$ is a Ricci scalar, and

$$
\begin{aligned}
& \mathcal{L}_{4}=G_{4}(\phi, X) R+G_{4, X}\left[(\square \phi)^{2}-\left(\nabla_{\mu} \nabla_{\nu} \phi\right)\left(\nabla^{\mu} \nabla^{\nu} \phi\right)\right], \\
& \mathcal{L}_{5}=G_{5}(\phi, X) G_{\mu \nu}\left(\nabla^{\mu} \nabla^{\nu} \phi\right)-\frac{1}{6} G_{5, X}\left[(\square \phi)^{3}-3(\square \phi)\left(\nabla_{\mu} \nabla_{\nu} \phi\right)\left(\nabla^{\mu} \nabla^{\nu} \phi\right)+2\left(\nabla^{\mu} \nabla_{\alpha} \phi\right)\left(\nabla^{\alpha} \nabla_{\beta} \phi\right)\left(\nabla^{\beta} \nabla_{\mu} \phi\right)\right],
\end{aligned}
$$

where $G_{i}(i=3,4,5)$ are functions in terms of $\phi$ and $X=-\partial^{\mu} \phi \partial_{\mu} \phi / 2$ with the partial derivatives $G_{i, X} \equiv \partial G_{i} / \partial X$, and $G_{\mu \nu}=R_{\mu \nu}-g_{\mu \nu} R / 2$ is the Einstein tensor $\left(R_{\mu \nu}\right.$ is the Ricci tensor). The action (1) is equivalent to that derived by Hordenski [42, 43]. In Ref. [43] the spectra of scalar and tensor perturbations were derived for the above models.

In this paper we compute the three-point correlation function of scalar metric perturbations for the inflationary models described by the action (1). Note that the calculation of scalar non-Gaussianities up to the term $G_{3}(\phi, X) \square \phi$ was carried out in Refs. [44 46] (see also Ref. [47] for non-minimal coupling models with $F(\phi) R$ ). Taking into account the Lagrangians $\mathcal{L}_{4}$ and $\mathcal{L}_{5}$, we can cover a wide variety of gravitational theories such as scalar-tensor theories 48] $\left(G_{4}=F(\phi)\right)$, field derivative couplings with gravity [49, 50] $\left(G_{5}=G(\phi)\right)$, Galileon gravity [29, 30] $\left(G_{3} \propto X, G_{4} \propto X^{2}\right.$, $\left.G_{5} \propto X^{2}\right)$, higher-curvature gravity [51] (including Gauss-Bonnet and $f(R)$ theories), $\alpha^{\prime}$ corrections appearing in lowenergy effective string theory [52].

Even in the presence of the new contributions coming from the terms $G_{4}$ and $G_{5}$ we shall show that the shape of non-Gaussianities can be well approximated by the equilateral type. The equilateral non-linear parameter $f_{\mathrm{NL}}^{\text {equil }}$ is derived on the quasi de Sitter background. We also apply our formula to concrete inflationary models in order to present the cases in which large non-Gaussianities can be realized. 
This paper is organized as follows. In Sec. II we derive the background equations of motion on the flat FriedmannLemaître-Robertson-Walker (FLRW) background and introduce a number of "slow-variation" parameters convenient for the calculation of primordial non-Gaussianities. In Sec. III the action (1) is expanded at second-order in perturbations in order to obtain the power spectrum of curvature perturbations generated during inflation. We also obtain the spectrum of gravitational waves and the tensor-to-scalar ratio. In Sec. IV we expand the action (1) at third-order in perturbations and compute the three-point correlation function of curvature perturbations. In Sec. $\mathrm{V}$ we study the shape of non-Gaussianities for the new terms appearing in the three-point correlation function. In Sec. VI we derive an explicit formula for the equilateral non-linear parameter $f_{\mathrm{NL}}^{\text {equil }}$ under the slow-variation approximation. In Sec. VII our formula for $f_{\mathrm{NL}}^{\text {equil }}$ is applied to a number of concrete models of inflation. Sec. VIII is devoted to conclusions.

\section{BACKGROUND EQUATIONS}

We first derive the background equations for the theories given by the action (11) on the flat FLRW background with the scale factor $a(t)$, where $t$ is cosmic time. Taking variation of the action at first order with respect to the metric elements $g_{00}, g_{i i}$, and the field $\phi$, where $g_{00}$ corresponds to the Lapse function $N$, we find

$$
\begin{aligned}
E_{1} \equiv & 3 M_{\mathrm{pl}}^{2} H^{2} F+P+6 H G_{4, \phi} \dot{\phi}+\left(G_{3, \phi}-12 H^{2} G_{4, X}+9 H^{2} G_{5, \phi}-P_{, X}\right) \dot{\phi}^{2} \\
& +\left(6 G_{4, \phi X}-3 G_{3, X}-5 G_{5, X} H^{2}\right) H \dot{\phi}^{3}+3\left(G_{5, \phi X}-2 G_{4, X X}\right) H^{2} \dot{\phi}^{4}-H^{3} G_{5, X X} \dot{\phi}^{5}=0, \\
E_{2} \equiv & 2\left[\left(G_{5, \phi}-2 G_{4, X}\right) \dot{\phi}^{2}-H G_{5, X} \dot{\phi}^{3}+F M_{\mathrm{pl}}^{2}\right] \dot{H}+3 M_{\mathrm{pl}}^{2} H^{2} F+P+4 H G_{4, \phi} \dot{\phi} \\
& +\left[2 G_{4, \phi}+4 H\left(G_{5, \phi}-G_{4, X}\right) \dot{\phi}+\left(2 G_{4, \phi X}-G_{3, X}-3 H^{2} G_{5, X}\right) \dot{\phi}^{2}+2 H\left(G_{5, \phi X}-2 G_{4, X X}\right) \dot{\phi}^{3}-H^{2} G_{5, X X} \dot{\phi}^{4}\right] \ddot{\phi} \\
& +\left(2 G_{4, \phi \phi}+3 H^{2} G_{5, \phi}-G_{3, \phi}-6 H^{2} G_{4, X}\right) \dot{\phi}^{2}+2 H\left(G_{5, \phi \phi}-G_{5, X} H^{2}-2 G_{4, \phi X}\right) \dot{\phi}^{3}-H^{2} G_{5, \phi X} \dot{\phi}^{4}=0,(5) \\
E_{3} \equiv & {\left[6 G_{4, \phi}+12\left(G_{5, \phi}-G_{4, X}\right) H \dot{\phi}+3\left(2 G_{4, \phi X}-G_{3, X}-3 H^{2} G_{5, X}\right) \dot{\phi}^{2}+6\left(G_{5, \phi X}-2 G_{4, X X}\right) H \dot{\phi}^{3}-3 H^{2} G_{5, X X} \dot{\phi}^{4}\right] \dot{H} } \\
& +\left\{3\left(G_{5, \phi X X}-2 G_{4, X X X}\right) H^{2} \dot{\phi}^{4}-H^{3} G_{5, X X X} \dot{\phi}^{5}+\left[3\left(2 G_{4, \phi X X}-G_{3, X X}\right)-7 H^{2} G_{5, X X}\right] H \dot{\phi}^{3}+2 G_{3, \phi}-P_{, X}\right. \\
& \left.+\left[3\left(5 G_{5, \phi X}-8 G_{4, X X}\right) H^{2}+G_{3, \phi X}-P_{, X X}\right] \dot{\phi}^{2}+6\left(3 G_{4, \phi X}-G_{5, X} H^{2}-G_{3, X}\right) H \dot{\phi}+6\left(G_{5, \phi}-G_{4, X}\right) H^{2}\right\} \ddot{\phi} \\
& +3\left(G_{5, \phi \phi X}-H^{2} G_{5, X X}-2 G_{4, \phi X X}\right) H^{2} \dot{\phi}^{4}+\left[\left(7 G_{5, \phi X}-18 G_{4, X X}\right) H^{2}+3\left(2 G_{4, \phi \phi X}-G_{3, \phi X}\right)\right] H \dot{\phi}^{3} \\
& +\left[3\left(G_{5, \phi \phi}+6 G_{4, \phi X}-3 G_{3, X}\right) H^{2}-9 H^{4} G_{5, X}-P_{, \phi X}+G_{3, \phi \phi}\right] \dot{\phi}^{2} \\
& +3\left[6\left(G_{5, \phi}-G_{4, X}\right) H^{2}+2 G_{3, \phi}-P_{, X}\right] H \dot{\phi}-H^{3} G_{5, \phi X X} \dot{\phi}^{5}+12 H^{2} G_{4, \phi}+P_{, \phi}=0,
\end{aligned}
$$

where $H=\dot{a} / a$ is the Hubble parameter, and

$$
F=1+\frac{2 G_{4}}{M_{\mathrm{pl}}^{2}} .
$$

Note that a dot represents a derivative with respect to $t$, whereas a comma corresponds to a derivative in terms of $\phi$ or $X$ (e.g., $\left.G_{5, \phi X}=\partial^{2} G_{5} / \partial X \partial \phi\right)$. These equations, because of the Bianchi identities, are not independent as one can directly verify that

$$
\dot{\phi} E_{3}-\dot{E}_{1}-3 H\left(E_{1}-E_{2}\right)=0 \text {. }
$$

Eliminating the terms $P$ from Eqs. (4) and (5), it follows that

$$
\begin{aligned}
\left(1-4 \delta_{G 4 X}-2 \delta_{G 5 X}+2 \delta_{G 5 \phi}\right) \epsilon= & \delta_{P X}+3 \delta_{G 3 X}-2 \delta_{G 3 \phi}+6 \delta_{G 4 X}-\delta_{G 4 \phi}-6 \delta_{G 5 \phi}+3 \delta_{G 5 X}+12 \delta_{G 4 X X}+2 \delta_{G 5 X X} \\
& -10 \delta_{G 4 \phi X}+2 \delta_{G 4 \phi \phi}-8 \delta_{G 5 \phi X}+2 \delta_{G 5 \phi \phi}-\delta_{\phi}\left(\delta_{G 3 X}+4 \delta_{G 4 X}-\delta_{G 4 \phi}\right. \\
& \left.+8 \delta_{G 4 X X}+3 \delta_{G 5 X}-4 \delta_{G 5 \phi}+2 \delta_{G 5 X X}-2 \delta_{G 4 \phi X}-4 \delta_{G 5 \phi X}\right)
\end{aligned}
$$

where we have defined the slow-variation parameters

$$
\begin{aligned}
& \epsilon=-\frac{\dot{H}}{H^{2}}, \quad \delta_{\phi}=\frac{\ddot{\phi}}{H \dot{\phi}}, \quad \delta_{P X}=\frac{P_{, X} X}{M_{\mathrm{pl}}^{2} H^{2} F}, \quad \delta_{G 3 X}=\frac{G_{3, X} \dot{\phi} X}{M_{\mathrm{pl}}^{2} H F}, \quad \delta_{G 3 \phi}=\frac{G_{3, \phi} X}{M_{\mathrm{pl}}^{2} H^{2} F}, \quad \delta_{G 4 X}=\frac{G_{4, X} X}{M_{\mathrm{pl}}^{2} F}, \\
& \delta_{G 4 \phi}=\frac{G_{4, \phi} \dot{\phi}}{M_{\mathrm{pl}}^{2} H F}, \quad \delta_{G 4 \phi X}=\frac{G_{4, \phi X} \dot{\phi} X}{M_{\mathrm{pl}}^{2} H F}, \quad \delta_{G 4 \phi \phi}=\frac{G_{4, \phi \phi} X}{M_{\mathrm{pl}}^{2} H^{2} F}, \quad \delta_{G 4 X X}=\frac{G_{4, X X} X^{2}}{M_{\mathrm{pl}}^{2} F}, \quad \delta_{G 5 \phi}=\frac{G_{5, \phi} X}{M_{\mathrm{pl}}^{2} F}, \\
& \delta_{G 5 X}=\frac{G_{5, X} H \dot{\phi} X}{M_{\mathrm{pl}}^{2} F}, \quad \delta_{G 5 X X}=\frac{G_{5, X X} H \dot{\phi} X^{2}}{M_{\mathrm{pl}}^{2} F} \quad \delta_{G 5 \phi X}=\frac{G_{5, \phi X} X^{2}}{M_{\mathrm{pl}}^{2} F}, \quad \delta_{G 5 \phi \phi}=\frac{G_{5, \phi \phi} \dot{\phi} X}{M_{\mathrm{pl}}^{2} H F} .
\end{aligned}
$$


Since $\epsilon \ll 1$ during inflation, we require that the slow-variation parameters defined above are much smaller than the order of unity.

Taking the time-derivative of the quantity $\delta_{G 4 X}$, we obtain the first-order quantity

$$
\eta_{G 4 X} \equiv \frac{\dot{\delta}_{G 4 X}}{H \delta_{G 4 X}}=\frac{2 \delta_{G 4 X X} \delta_{\phi}}{\delta_{G 4 X}}+\frac{\delta_{G 4 \phi X}}{\delta_{G 4 X}}+2 \delta_{\phi}-\delta_{F},
$$

where $\delta_{F} \equiv \dot{F} /(H F)$. This means that $\delta_{G 4 \phi X}$ is higher than the first order. Likewise one can show that

$$
\left\{\delta_{G 3 \phi X}, \delta_{G 3 \phi \phi}, \delta_{G 4 \phi X}, \delta_{G 4 \phi \phi}, \delta_{G 5 \phi X}, \delta_{G 5 \phi \phi}\right\}=\mathcal{O}\left(\epsilon^{2}\right)
$$

where

$$
\delta_{G 3 \phi X}=\frac{G_{3, \phi X} X^{2}}{M_{\mathrm{pl}}^{2} H^{2} F}, \quad \delta_{G 3 \phi \phi}=\frac{G_{3, \phi \phi} \dot{\phi} X}{M_{\mathrm{pl}}^{2} H^{3} F} .
$$

Then, at first order, Eq. (9) reduces to

$$
\epsilon \simeq \delta_{P X}+3 \delta_{G 3 X}-2 \delta_{G 3 \phi}+6 \delta_{G 4 X}-\delta_{G 4 \phi}-6 \delta_{G 5 \phi}+3 \delta_{G 5 X}+12 \delta_{G 4 X X}+2 \delta_{G 5 X X} .
$$

It is also convenient to notice the following relation

$$
\delta_{F}=2 \delta_{G 4 \phi}+4 \delta_{G 4 X} \delta_{\phi},
$$

that we will use hereafter.

\section{SECOND-ORDER ACTION}

In order to discuss the primordial non-Gaussianities we need to first study linear perturbation theory. We shall use the momentum and Hamiltonian constraints to integrate out all the auxiliary fields. For the calculation of scalar non-Gaussianities it is convenient to choose the ADM metric [53] in the form

$$
d s^{2}=-\left[(1+\alpha)^{2}-a(t)^{-2} e^{-2 \mathcal{R}}(\partial \psi)^{2}\right] d t^{2}+2 \partial_{i} \psi d t d x^{i}+a(t)^{2} e^{2 \mathcal{R}} d \boldsymbol{x}^{2},
$$

where $\alpha, \psi$, and $\mathcal{R}$ are scalar metric perturbations [11]. We choose the uniform field gauge, $\delta \phi=0$, to fix the time-component of a gauge-transformation vector $\xi^{\mu}$. The spatial part of $\xi^{\mu}$ is fixed by gauging away a perturbation $E$ that appears as a form $E_{, i j}$ in the metric (16) [54].

Expanding the action (10) up to second order in the perturbations, we find the following result

$$
S_{2}=\int d t d^{3} x a^{3}\left[-3 w_{1} \dot{\mathcal{R}}^{2}+\frac{1}{a^{2}}\left(2 w_{1} \dot{\mathcal{R}}-w_{2} \alpha\right) \partial^{2} \psi-\frac{2 w_{1}}{a^{2}} \alpha \partial^{2} \mathcal{R}+3 w_{2} \alpha \dot{\mathcal{R}}+\frac{1}{3} w_{3} \alpha^{2}+\frac{w_{4}}{a^{2}}(\partial \mathcal{R})^{2}\right]
$$

where

$$
\begin{aligned}
w_{1}= & M_{\mathrm{pl}}^{2} F-4 X G_{4, X}-2 H X \dot{\phi} G_{5, X}+2 X G_{5, \phi} \\
w_{2}= & 2 M_{\mathrm{pl}}^{2} H F-2 X \dot{\phi} G_{3, X}-16 H\left(X G_{4, X}+X^{2} G_{4, X X}\right)+2 \dot{\phi}\left(G_{4, \phi}+2 X G_{4, \phi X}\right) \\
& -2 H^{2} \dot{\phi}\left(5 X G_{5, X}+2 X^{2} G_{5, X X}\right)+4 H X\left(3 G_{5, \phi}+2 X G_{5, \phi X}\right) \\
w_{3}= & -9 M_{\mathrm{pl}}^{2} H^{2} F+3\left(X P_{, X}+2 X^{2} P_{, X X}\right)+18 H \dot{\phi}\left(2 X G_{3, X}+X^{2} G_{3, X X}\right)-6 X\left(G_{3, \phi}+X G_{3, \phi X}\right) \\
& +18 H^{2}\left(7 X G_{4, X}+16 X^{2} G_{4, X X}+4 X^{3} G_{4, X X X}\right)-18 H \dot{\phi}\left(G_{4, \phi}+5 X G_{4, \phi X}+2 X^{2} G_{4, \phi X X}\right) \\
& +6 H^{3} \dot{\phi}\left(15 X G_{5, X}+13 X^{2} G_{5, X X}+2 X^{3} G_{, 5 X X X}\right)-18 H^{2} X\left(6 G_{5, \phi}+9 X G_{5, \phi X}+2 X^{2} G_{5, \phi X X}\right) \\
w_{4}= & M_{\mathrm{pl}}^{2} F-2 X G_{5, \phi}-2 X G_{5, X} \ddot{\phi} .
\end{aligned}
$$

Although the coefficients are quite complicated, the structure of the action (17) is similar to that found in Ref. [45]. It is straightforward to find the momentum and Hamiltonian constraints

$$
\begin{aligned}
\alpha & =L_{1} \dot{\mathcal{R}}, \\
\frac{1}{a^{2}} \partial^{2} \psi & =\frac{2 w_{3}}{3 w_{2}} \alpha+3 \dot{\mathcal{R}}-\frac{2 w_{1}}{w_{2}} \frac{1}{a^{2}} \partial^{2} \mathcal{R},
\end{aligned}
$$


where

$$
L_{1}=\frac{2 w_{1}}{w_{2}}
$$

Substituting Eq. (22) into Eq. (17) and making integrations by parts, the second-order action reduces to the following form

$$
S_{2}=\int d t d^{3} x a^{3} Q\left[\dot{\mathcal{R}}^{2}-\frac{c_{s}^{2}}{a^{2}}(\partial \mathcal{R})^{2}\right]
$$

where

$$
\begin{aligned}
Q & =\frac{w_{1}\left(4 w_{1} w_{3}+9 w_{2}^{2}\right)}{3 w_{2}^{2}} \\
c_{s}^{2} & =\frac{3\left(2 w_{1}^{2} w_{2} H-w_{2}^{2} w_{4}+4 w_{1} \dot{w}_{1} w_{2}-2 w_{1}^{2} \dot{w}_{2}\right)}{w_{1}\left(4 w_{1} w_{3}+9 w_{2}^{2}\right)}
\end{aligned}
$$

In order to avoid the appearance of ghosts and Laplacian instabilities we require the conditions

$$
Q>0, \quad c_{s}^{2}>0
$$

For later convenience we introduce the following parameter

$$
\epsilon_{s} \equiv \frac{Q c_{s}^{2}}{M_{\mathrm{pl}}^{2} F}=\frac{2 w_{1}^{2} w_{2} H-w_{2}^{2} w_{4}+4 w_{1} \dot{w}_{1} w_{2}-2 w_{1}^{2} \dot{w}_{2}}{M_{\mathrm{pl}}^{2} F w_{2}^{2}}
$$

Expansion of $\epsilon_{s}$ in terms of the slow-variation parameters leads to

$$
\begin{aligned}
\epsilon_{s} & =\epsilon+\delta_{G 3 X}+\delta_{G 4 \phi}+8 \delta_{G 4 X X}+\delta_{G 5 X}+2 \delta_{G 5 X X}+\mathcal{O}\left(\epsilon^{2}\right) \\
& =\delta_{P X}+4 \delta_{G 3 X}-2 \delta_{G 3 \phi}+6 \delta_{G 4 X}+20 \delta_{G 4 X X}+4 \delta_{G 5 X}+4 \delta_{G 5 X X}-6 \delta_{G 5 \phi}+\mathcal{O}\left(\epsilon^{2}\right),
\end{aligned}
$$

where we have used Eqs. (12), (14), and (15).

The two-point correlation function of curvature perturbations $\mathcal{R}$ can be derived by employing the standard method of quantizing the fields on a de Sitter background [54]. The power spectrum of $\mathcal{R}$, some time after the Hubble radius crossing, is given by

$$
\mathcal{P}_{\mathcal{R}}=\frac{H^{2}}{8 \pi^{2} Q c_{s}^{3}}=\frac{H^{2}}{8 \pi^{2} M_{\mathrm{pl}}^{2} F \epsilon_{s} c_{s}} .
$$

Its spectral index, evaluated at $c_{s} k=a H$ ( $k$ is a comoving wave number), can be derived as follows

$$
\begin{aligned}
n_{\mathcal{R}}-1 & =\left.\frac{d \ln \mathcal{P}_{\mathcal{R}}}{d \ln k}\right|_{c_{s} k=a H} \\
& =-2 \epsilon-\delta_{F}-\eta_{s}-s=-2 \epsilon_{s}-\eta_{s}-s+2 \delta_{G 3 X}+16 \delta_{G 4 X X}+2 \delta_{G 5 X}+4 \delta_{G 5 X X}+\mathcal{O}\left(\epsilon^{2}\right),
\end{aligned}
$$

where

$$
\eta_{s}=\frac{\dot{\epsilon}_{s}}{H \epsilon_{s}}, \quad s=\frac{\dot{c}_{s}}{H c_{s}}
$$

Here we have assumed that $c_{s}$ is a slowly varying function, such that $|s| \ll 1$.

Let us now proceed to the power spectrum of the gravitational waves for the theories under consideration. We study the tensor perturbations of the form

$$
d s^{2}=-d t^{2}+a^{2}(t)\left(\delta_{i j}+h_{i j}^{T T}\right) d x^{i} d x^{j},
$$

where $h_{i j}^{T T}$ is transverse and traceless. It is known that the $h_{i j}^{T T}$ can be decomposed into two independent polarization modes, namely $h_{i j}^{T T}=h_{+} e_{i j}^{+}+h_{\times} e_{i j}^{\times}$. We choose the normalization for the two matrices such that, in Fourier 
space, $e_{i j}^{\lambda}(\boldsymbol{k}) e_{i j}^{\lambda}(-\boldsymbol{k})^{*}=2$, (where $\left.\lambda=+, \times\right)$, and $e_{i j}^{+}(\boldsymbol{k}) e_{i j}^{\times}(-\boldsymbol{k})^{*}=0$. In this case the second-order action for the gravitational waves can be written as

$$
S=\sum_{\lambda} \int d t d^{3} x a^{3} Q_{T}\left[\dot{h}_{\lambda}^{2}-\frac{c_{T}^{2}}{a^{2}}\left(\partial h_{\lambda}\right)^{2}\right],
$$

where

$$
\begin{aligned}
Q_{T} & =\frac{w_{1}}{4}=\frac{1}{4} M_{\mathrm{pl}}^{2} F\left(1-4 \delta_{G 4 X}-2 \delta_{G 5 X}+2 \delta_{G 5 \phi}\right), \\
c_{T}^{2} & =\frac{w_{4}}{w_{1}}=1+4 \delta_{G 4 X}+2 \delta_{G 5 X}-4 \delta_{G 5 \phi}+\mathcal{O}\left(\epsilon^{2}\right) .
\end{aligned}
$$

Provided that $\left\{\left|\delta_{G 4 X}\right|,\left|\delta_{G 5 X}\right|,\left|\delta_{G 5 \phi}\right|\right\} \ll 1$, one has $Q_{T} \simeq M_{\mathrm{pl}}^{2} F / 4$ and hence the no-ghost condition $Q_{T}>0$ is satisfied for $F>0$. Since $c_{T}^{2}$ is close to 1 , there are no Laplacian instabilities for tensor perturbations.

The spectrum of tensor perturbations is given by

$$
\mathcal{P}_{T}=\frac{H^{2}}{2 \pi^{2} Q_{T} c_{T}^{3}} \simeq \frac{2 H^{2}}{\pi^{2} M_{\mathrm{pl}}^{2} F},
$$

together with the spectral index

$$
\begin{aligned}
n_{T} & =\left.\frac{d \ln \mathcal{P}_{T}}{d \ln k}\right|_{c_{T} k=a H} \\
& =-2 \epsilon-\delta_{F}=-2 \epsilon_{s}+2 \delta_{G 3 X}+16 \delta_{G 4 X X}+2 \delta_{G 5 X}+4 \delta_{G 5 X X}+\mathcal{O}\left(\epsilon^{2}\right) .
\end{aligned}
$$

For those times during inflation when both $\mathcal{P}_{T}$ and $\mathcal{P}_{\mathcal{R}}$ remain approximately constant, the tensor-to-scalar ratio can be estimated as

$$
r=\frac{\mathcal{P}_{T}}{\mathcal{P}_{\mathcal{R}}} \simeq 16 c_{s} \epsilon_{s}
$$

Then we have the consistency relation

$$
r \simeq 8 c_{s}\left(-n_{T}+2 \delta_{G 3 X}+16 \delta_{G 4 X X}+2 \delta_{G 5 X}+4 \delta_{G 5 X X}\right) .
$$

The consistency relation in standard inflation $\left(r=-8 c_{s} n_{T}\right)$ is modified because of the presence of the terms $G_{i}$ $(i=3,4,5)$.

The spectra of scalar and tensor perturbations coincide with those derived in Ref. [43].

\section{THREE-POINT CORRELATION FUNCTION}

We proceed to the calculation of the three-point correlation function of curvature perturbations for the theories described by the action (1). In doing so we expand this action up to third order in perturbations [11]. Although the calculation is quite involved, the steps for the derivation of the three-point correlation function are similar to those given in detail in Ref. [45]. The third-order action is given by

$$
\begin{aligned}
S_{3}= & \int d t d^{3} x a^{3}\left\{a_{1} \alpha^{3}+\alpha^{2}\left(a_{2} \mathcal{R}+a_{3} \dot{\mathcal{R}}+a_{4} \partial^{2} \mathcal{R} / a^{2}+a_{5} \partial^{2} \psi / a^{2}\right)\right. \\
& +\alpha\left[a_{6} \partial_{i} \mathcal{R} \partial_{i} \psi / a^{2}+a_{7} \dot{\mathcal{R}} \mathcal{R}+a_{8} \dot{\mathcal{R}} \partial^{2} \mathcal{R} / a^{2}+a_{9}\left(\partial_{i} \partial_{j} \psi \partial_{i} \partial_{j} \psi-\partial^{2} \psi \partial^{2} \psi\right) / a^{4}\right. \\
& \left.+a_{10}\left(\partial_{i} \partial_{j} \psi \partial_{i} \partial_{j} \mathcal{R}-\partial^{2} \psi \partial^{2} \mathcal{R}\right) / a^{4}+a_{11} \mathcal{R} \partial^{2} \psi / a^{2}+a_{12} \dot{\mathcal{R}} \partial^{2} \psi / a^{2}+a_{13} \mathcal{R} \partial^{2} \mathcal{R} / a^{2}+a_{14}(\partial \mathcal{R})^{2} / a^{2}+a_{15} \dot{\mathcal{R}}^{2}\right] \\
& +b_{1} \dot{\mathcal{R}}^{3}+b_{2} \mathcal{R}(\partial \mathcal{R})^{2} / a^{2}+b_{3} \dot{\mathcal{R}}^{2} \mathcal{R}+c_{1} \dot{\mathcal{R}} \partial_{i} \mathcal{R} \partial_{i} \psi / a^{2}+c_{2} \dot{\mathcal{R}}^{2} \partial^{2} \psi / a^{2}+c_{3} \dot{\mathcal{R}} \mathcal{R} \partial^{2} \psi / a^{2} \\
& \left.+\left(d_{1} \dot{\mathcal{R}}+d_{2} \mathcal{R}\right)\left(\partial_{i} \partial_{j} \psi \partial_{i} \partial_{j} \psi-\partial^{2} \psi \partial^{2} \psi\right) / a^{4}+d_{3} \partial_{i} \mathcal{R} \partial_{i} \psi \partial^{2} \psi / a^{4}\right\}
\end{aligned}
$$


where

$$
\begin{aligned}
a_{1}= & 3 M_{\mathrm{pl}}^{2} H^{2} F-\left(X P_{, X}+4 X^{2} P_{, X X}+4 X^{3} P_{, X X X} / 3\right)-2 H \dot{\phi}\left(10 X G_{3, X}+11 X^{2} G_{3, X X}+2 X^{3} G_{3, X X X}\right) \\
& +2 X\left(G_{3, \phi}+7 X G_{3, \phi X} / 3+2 X^{2} G_{3, \phi X X} / 3\right)-2 H^{2}\left(33 X G_{4, X}+126 X^{2} G_{4, X X}+68 X^{3} G_{4, X X X}+8 X^{4} G_{4, X X X X}\right) \\
& +2 H \dot{\phi}\left(3 G_{4, \phi}+27 X G_{4, \phi X}+24 X^{2} G_{4, \phi X X}+4 X^{3} G_{4, \phi X X X}\right)-H^{3} \dot{\phi}\left(70 X G_{5, X}+98 X^{2} G_{5, X X}+32 X^{3} G_{5, X X X}\right. \\
& \left.+8 X^{4} G_{5, X X X X} / 3\right)+2 H^{2} X\left(30 G_{5, \phi}+75 X G_{5, \phi X}+36 X^{2} G_{5, \phi X X}+4 X^{3} G_{5, \phi X X X}\right), \\
a_{2}= & w_{3}, \\
a_{3}= & -3 a_{5} \\
= & -6 M_{\mathrm{pl}}^{2} H F+6 \dot{\phi}\left(2 X G_{3, X}+X^{2} G_{3, X X}\right)+12 H\left(7 X G_{4, X}+16 X^{2} G_{4, X X}+4 X^{3} G_{4, X X X}\right) \\
& -6 \dot{\phi}\left(G_{4, \phi}+5 X G_{4, \phi X}+2 X^{2} G_{4, \phi X X}\right)+6 H^{2} \dot{\phi}\left(15 X G_{5, X}+13 X^{2} G_{5, X X}+2 X^{3} G_{5, X X X}\right) \\
& -12 H X\left(6 G_{5, \phi}+9 X G_{5, \phi X}+2 X^{2} G_{5, \phi X X}\right), \\
a_{4}= & -4\left(X G_{4, X}+2 X^{2} G_{4, X X}\right)-8 H \dot{\phi}\left(X G_{5, X}+X^{2} G_{5, X X} / 2\right)+4 X\left(G_{5, \phi}+2 X G_{5, \phi X}\right), \\
a_{6}= & -a_{7} / 9=a_{11}=-w_{2}, \\
a_{8}= & 2 a_{10}=2 b_{1}=-2 c_{2}=-4 d_{1}=4 X \dot{\phi} G_{5, X}, \\
a_{9}= & a_{12} / 4=-a_{15} / 6 \\
= & \left.-M_{\mathrm{pl}}^{2} F / 2+4\left(X G_{4, X}+X^{2} G_{4, X X}\right)+H \dot{\phi}\left(5 X G_{5, X}+2 X^{2} G_{5, X X}\right)-X_{\left(3 G_{5, \phi}\right.}+2 X G_{5, \phi X}\right), \\
a_{13}= & 2 a_{14}=2 b_{3} / 9=-c_{1}=-c_{3}=-4 d_{2} / 3=d_{3}=-2 w_{1}, \\
b_{2}= & w_{4} .
\end{aligned}
$$

We use Eq. (22) to eliminate the field $\alpha$ from the action (42), which gives

$$
\begin{aligned}
S_{3}= & \int d t d^{3} x a^{3}\left\{A_{1} \dot{\mathcal{R}}^{3}+A_{2} \dot{\mathcal{R}}^{2} \partial^{2} \mathcal{R} / a^{2}+A_{3} \dot{\mathcal{R}}^{2} \partial^{2} \psi / a^{2}+A_{4} \mathcal{R} \dot{\mathcal{R}}^{2}+\left(A_{5} \dot{\mathcal{R}}+A_{6} \mathcal{R}\right)\left(\partial_{i} \partial_{j} \psi \partial_{i} \partial_{j} \psi-\partial^{2} \psi \partial^{2} \psi\right) / a^{4}\right. \\
& \left.+A_{7} \dot{\mathcal{R}}\left(\partial_{i} \partial_{j} \psi \partial_{i} \partial_{j} \mathcal{R}-\partial^{2} \psi \partial^{2} \mathcal{R}\right) / a^{4}+A_{8} \mathcal{R}(\partial \mathcal{R})^{2} / a^{2}+A_{9} \partial_{i} \mathcal{R} \partial_{i} \psi \partial^{2} \psi / a^{4}\right\}
\end{aligned}
$$

where

$$
\begin{array}{lll}
A_{1}=b_{1}+L_{1} a_{15}+L_{1}^{2} a_{3}+L_{1}^{3} a_{1}, & A_{2}=L_{1}\left(L_{1} a_{4}+a_{8}\right), & A_{3}=c_{2}+L_{1} a_{12}+L_{1}^{2} a_{5}, \\
A_{4}=b_{3}+L_{1} a_{7}+L_{1}^{2} a_{2}=3 Q, & A_{5}=L_{1} a_{9}+d_{1}, & A_{6}=d_{2}, \\
A_{7}=L_{1} a_{10}, & A_{8}=b_{2}+a_{13} \dot{L}_{1} / 2+L_{1}\left(\dot{a}_{13}+H a_{13}\right) / 2, & A_{9}=d_{3} .
\end{array}
$$

The next step is to eliminate the field $\psi$ by using Eq. (23). Introducing an auxiliary field $\mathcal{X}$ satisfying the relation

$$
\psi=-L_{1} \mathcal{R}+\frac{a^{2} \mathcal{X}}{w_{1}},
$$

it follows that $\partial^{2} \mathcal{X}=Q \dot{\mathcal{R}}$ from Eq. (23). Plugging Eq. (54) into the action (52), we obtain

$$
S_{3}=\int d t d^{3} x\left(a^{3} f_{1}+a f_{2}+f_{3} / a\right),
$$

where

$$
\begin{aligned}
f_{1} \equiv & \left(A_{1}+A_{3} \frac{Q}{w_{1}}-A_{5} \frac{Q^{2}}{w_{1}^{2}}\right) \dot{\mathcal{R}}^{3}+\left(A_{4}-A_{6} \frac{Q^{2}}{w_{1}^{2}}\right) \mathcal{R} \dot{\mathcal{R}}^{2}+A_{9} \frac{Q}{w_{1}^{2}} \dot{\mathcal{R}} \partial_{i} \mathcal{R} \partial_{i} \mathcal{X} \\
& +\frac{1}{w_{1}^{2}}\left(A_{5} \dot{\mathcal{R}}+A_{6} \mathcal{R}\right)\left(\partial_{i} \partial_{j} \mathcal{X}\right)\left(\partial_{i} \partial_{j} \mathcal{X}\right) \\
f_{2} \equiv & \left(A_{2}-A_{3} L_{1}+A_{5} \frac{2 L_{1} Q}{w_{1}}-A_{7} \frac{Q}{w_{1}}\right) \dot{\mathcal{R}}^{2} \partial^{2} \mathcal{R}+A_{6} \frac{2 L_{1} Q}{w_{1}} \mathcal{R} \dot{\mathcal{R}} \partial^{2} \mathcal{R}+A_{8} \mathcal{R}(\partial \mathcal{R})^{2}-A_{9} \frac{L_{1} Q}{w_{1}} \dot{\mathcal{R}}(\partial \mathcal{R})^{2} \\
& +\frac{A_{7}-2 A_{5} L_{1}}{w_{1}} \dot{\mathcal{R}}\left(\partial_{i} \partial_{j} \mathcal{R}\right)\left(\partial_{i} \partial_{j} \mathcal{X}\right)-\frac{2 A_{6} L_{1}}{w_{1}} \mathcal{R}\left(\partial_{i} \partial_{j} \mathcal{R}\right)\left(\partial_{i} \partial_{j} \mathcal{X}\right)-\frac{A_{9} L_{1}}{w_{1}} \partial^{2} \mathcal{R} \partial_{i} \mathcal{R} \partial_{i} \mathcal{X} \\
f_{3} \equiv & \left(A_{5} L_{1}^{2}-A_{7} L_{1}\right) \dot{\mathcal{R}}\left[\left(\partial_{i} \partial_{j} \mathcal{R}\right)\left(\partial_{i} \partial_{j} \mathcal{R}\right)-\left(\partial^{2} \mathcal{R}\right)^{2}\right]+A_{6} L_{1}^{2} \mathcal{R}\left[\left(\partial_{i} \partial_{j} \mathcal{R}\right)\left(\partial_{i} \partial_{j} \mathcal{R}\right)-\left(\partial^{2} \mathcal{R}\right)^{2}\right]+A_{9} L_{1}^{2}(\partial \mathcal{R})^{2} \partial^{2} \mathcal{R} .(58
\end{aligned}
$$


Along the same lines of Ref. [45, 55] we express the action (55) in a more simple form by carrying out many integrations by parts. Finally we reach the following action

$$
S_{3}=\int d t \mathcal{L}_{3}
$$

where

$$
\begin{aligned}
\mathcal{L}_{3}= & \int d^{3} x\left\{a^{3} \mathcal{C}_{1} M_{\mathrm{pl}}^{2} \mathcal{R} \dot{\mathcal{R}}^{2}+a \mathcal{C}_{2} M_{\mathrm{pl}}^{2} \mathcal{R}(\partial \mathcal{R})^{2}+a^{3} \mathcal{C}_{3} M_{\mathrm{pl}} \dot{\mathcal{R}}^{3}+a^{3} \mathcal{C}_{4} \dot{\mathcal{R}}\left(\partial_{i} \mathcal{R}\right)\left(\partial_{i} \mathcal{X}\right)+a^{3}\left(\mathcal{C}_{5} / M_{\mathrm{pl}}^{2}\right) \partial^{2} \mathcal{R}(\partial \mathcal{X})^{2}\right. \\
& +a \mathcal{C}_{6} \dot{\mathcal{R}}^{2} \partial^{2} \mathcal{R}+\mathcal{C}_{7}\left[\partial^{2} \mathcal{R}(\partial \mathcal{R})^{2}-\mathcal{R} \partial_{i} \partial_{j}\left(\partial_{i} \mathcal{R}\right)\left(\partial_{j} \mathcal{R}\right)\right] / a+a\left(\mathcal{C}_{8} / M_{\mathrm{pl}}\right)\left[\partial^{2} \mathcal{R} \partial_{i} \mathcal{R} \partial_{i} \mathcal{X}-\mathcal{R} \partial_{i} \partial_{j}\left(\partial_{i} \mathcal{R}\right)\left(\partial_{j} \mathcal{X}\right)\right] \\
& \left.+\left.\mathcal{F}_{1} \frac{\delta \mathcal{L}_{2}}{\delta \mathcal{R}}\right|_{1}\right\}
\end{aligned}
$$

and the dimensionless coefficients $\mathcal{C}_{i}(i=1, \cdots, 8)$ are

$$
\begin{aligned}
& \mathcal{C}_{1}=\frac{1}{M_{\mathrm{pl}}^{2}}\left[3 Q+q_{1}(\dot{Q}+3 H Q)-Q \dot{q}_{1}\right], \\
& \mathcal{C}_{2}=\frac{1}{M_{\mathrm{pl}}^{2}}\left[A_{8}+\frac{1}{a} \frac{d}{d t}\left(a L_{1} Q\right)\right], \\
& \mathcal{C}_{3}=\frac{1}{M_{\mathrm{pl}}}\left(A_{1}+A_{3} \frac{Q}{w_{1}}-q_{1} Q\right), \\
& \mathcal{C}_{4}=\frac{Q}{w_{1}}\left[\frac{1}{w_{1}}\left(A_{6}+A_{9}\right)-w_{1} \frac{d}{d t}\left(\frac{A_{5}}{w_{1}^{2}}\right)+\frac{3 H A_{5}}{w_{1}}\right], \\
& \mathcal{C}_{5}=\frac{M_{\mathrm{pl}}^{2}}{2}\left[\frac{A_{6}}{w_{1}^{2}}-\frac{d}{d t}\left(\frac{A_{5}}{w_{1}^{2}}\right)+\frac{3 H A_{5}}{w_{1}^{2}}\right], \\
& \mathcal{C}_{6}=A_{2}-A_{3} L_{1}, \\
& \mathcal{C}_{7}=q_{3}-\frac{Q c_{s}^{2}}{2 w_{1}}\left(A_{7}-2 A_{5} L_{1}\right), \\
& \mathcal{C}_{8}=M_{\mathrm{pl}}\left(\frac{q_{2}}{2}-\frac{2 c_{s}^{2} A_{5} Q}{w_{1}^{2}}\right) .
\end{aligned}
$$

The terms $q_{1}, q_{2}$, and $q_{3}$ appear during the step to derive (59) from (55), and are given by [45]

$$
\begin{aligned}
& q_{1}=-\frac{L_{1}}{c_{s}^{2}} \\
& q_{2}=-\frac{4 A_{6} L_{1}}{w_{1}}-a^{2} \frac{d}{d t}\left(\frac{A_{7}-2 A_{5} L_{1}}{a^{2} w_{1}}\right)-\frac{2 A_{9} L_{1}}{w_{1}}, \\
& q_{3}=A_{6} L_{1}^{2}-\frac{a}{3} \frac{d}{d t}\left(\frac{A_{5} L_{1}^{2}-A_{7} L_{1}}{a}\right)+\frac{2}{3} A_{9} L_{1}^{2} .
\end{aligned}
$$

The last term in Eq. (60) is the product of the following quantities

$$
\mathcal{F}_{1}=\frac{A_{5}}{w_{1}^{2}}\left\{\left(\partial_{k} \mathcal{R}\right)\left(\partial_{k} \mathcal{X}\right)-\partial^{-2} \partial_{i} \partial_{j}\left[\left(\partial_{i} \mathcal{R}\right)\left(\partial_{j} \mathcal{X}\right)\right]\right\}+q_{1} \mathcal{R} \dot{\mathcal{R}}+\frac{A_{7}-2 A_{5} L_{1}}{4 w_{1} a^{2}}\left\{(\partial \mathcal{R})^{2}-\partial^{-2} \partial_{i} \partial_{j}\left[\left(\partial_{i} \mathcal{R}\right)\left(\partial_{j} \mathcal{R}\right)\right]\right\}
$$

and

$$
\left.\frac{\delta \mathcal{L}_{2}}{\delta \mathcal{R}}\right|_{1} \equiv-2\left[\frac{d}{d t}\left(a^{3} Q \dot{\mathcal{R}}\right)-a Q c_{s}^{2} \partial^{2} \mathcal{R}\right]
$$

The coefficient $\mathcal{F}_{1}$ includes only the time and spatial derivatives of $\mathcal{R}$ and $\mathcal{X}$. In the paper of Maldacena 11 the term $\mathcal{R}^{2}$ is also present in the expression of $\mathcal{F}_{1}$, which gives rise to the contribution of the order of the slow-variation parameter in the final expression of the non-linear parameter. After integrations by parts it is possible to move this contribution to other terms such as $\mathcal{C}_{1}$ [45], which we have done in Eq. (60). Hence, as in Refs. [56], we can neglect the contribution coming from the term (72). 
Defining the conformal time as $\tau=\int a^{-1} d t$, the vacuum expectation value of curvature perturbations for the three-point operator at $\tau=\tau_{f}$ is [11, 24, 57$]$

$$
\left\langle\mathcal{R}\left(\boldsymbol{k}_{1}\right) \mathcal{R}\left(\boldsymbol{k}_{2}\right) \mathcal{R}\left(\boldsymbol{k}_{3}\right)\right\rangle=-i \int_{\tau_{i}}^{\tau_{f}} d \tau a\left\langle 0\left|\left[\mathcal{R}\left(\tau_{f}, \boldsymbol{k}_{1}\right) \mathcal{R}\left(\tau_{f}, \boldsymbol{k}_{2}\right) \mathcal{R}\left(\tau_{f}, \boldsymbol{k}_{3}\right), \mathcal{H}_{\mathrm{int}}(\tau)\right]\right| 0\right\rangle,
$$

where the interacting Hamiltonian $\mathcal{H}_{\text {int }}$ is given by $\mathcal{H}_{\text {int }}=-\mathcal{L}_{3}$. Note that $\tau_{i}$ corresponds to the initial time at which the perturbations are deep inside the Hubble radius. One can take $\tau_{i} \rightarrow-\infty$ and $\tau_{f} \rightarrow 0$ because $\tau \simeq-1 /(a H)$ during inflation. When the integral with respect to $\tau$ is carried out, we treat the terms $\mathcal{C}_{i}(i=1, \cdots, 8)$ as constants because their variations are small relative to the scale factor $a$. Each contribution of the three-point correlation function coming from the terms $\mathcal{C}_{i}(i=1, \cdots, 8)$ is [45]

$$
\begin{aligned}
& \left\langle\mathcal{R}\left(\boldsymbol{k}_{1}\right) \mathcal{R}\left(\boldsymbol{k}_{2}\right) \mathcal{R}\left(\boldsymbol{k}_{3}\right)\right\rangle^{(1)}=(2 \pi)^{3} \delta^{(3)}\left(\boldsymbol{k}_{1}+\boldsymbol{k}_{2}+\boldsymbol{k}_{3}\right) \frac{\mathcal{C}_{1} M_{\mathrm{pl}}^{2} H^{4}}{16 Q^{3} c_{s}^{6}} \frac{1}{\left(k_{1} k_{2} k_{3}\right)^{3}}\left(\frac{k_{2}^{2} k_{3}^{2}}{K}+\frac{k_{1} k_{2}^{2} k_{3}^{2}}{K^{2}}+\mathrm{sym}\right), \\
& \left\langle\mathcal{R}\left(\boldsymbol{k}_{1}\right) \mathcal{R}\left(\boldsymbol{k}_{2}\right) \mathcal{R}\left(\boldsymbol{k}_{3}\right)\right\rangle^{(2)}=(2 \pi)^{3} \delta^{(3)}\left(\boldsymbol{k}_{1}+\boldsymbol{k}_{2}+\boldsymbol{k}_{3}\right) \frac{\mathcal{C}_{2} M_{\mathrm{pl}}^{2} H^{4}}{16 Q^{3} c_{s}^{8}} \frac{1}{\left(k_{1} k_{2} k_{3}\right)^{3}} \\
& \times\left[\left(\boldsymbol{k}_{1} \cdot \boldsymbol{k}_{2}+\boldsymbol{k}_{2} \cdot \boldsymbol{k}_{3}+\boldsymbol{k}_{3} \cdot \boldsymbol{k}_{1}\right)\left(-K+\frac{k_{1} k_{2}+k_{2} k_{3}+k_{3} k_{1}}{K}+\frac{k_{1} k_{2} k_{3}}{K^{2}}\right)\right], \\
& \left\langle\mathcal{R}\left(\boldsymbol{k}_{1}\right) \mathcal{R}\left(\boldsymbol{k}_{2}\right) \mathcal{R}\left(\boldsymbol{k}_{3}\right)\right\rangle^{(3)}=(2 \pi)^{3} \delta^{(3)}\left(\boldsymbol{k}_{1}+\boldsymbol{k}_{2}+\boldsymbol{k}_{3}\right) \frac{3 \mathcal{C}_{3} M_{\mathrm{pl}} H^{5}}{8 Q^{3} c_{s}^{6}} \frac{1}{k_{1} k_{2} k_{3}} \frac{1}{K^{3}}, \\
& \left\langle\mathcal{R}\left(\boldsymbol{k}_{1}\right) \mathcal{R}\left(\boldsymbol{k}_{2}\right) \mathcal{R}\left(\boldsymbol{k}_{3}\right)\right\rangle^{(4)}=(2 \pi)^{3} \delta^{(3)}\left(\boldsymbol{k}_{1}+\boldsymbol{k}_{2}+\boldsymbol{k}_{3}\right) \frac{\mathcal{C}_{4} H^{4}}{32 Q^{2} c_{s}^{6}} \frac{1}{\left(k_{1} k_{2} k_{3}\right)^{3}}\left[\frac{\left(\boldsymbol{k}_{1} \cdot \boldsymbol{k}_{2}\right) k_{3}^{2}}{K}\left(2+\frac{k_{1}+k_{2}}{K}\right)+\mathrm{sym}\right], \\
& \left\langle\mathcal{R}\left(\boldsymbol{k}_{1}\right) \mathcal{R}\left(\boldsymbol{k}_{2}\right) \mathcal{R}\left(\boldsymbol{k}_{3}\right)\right\rangle^{(5)}=(2 \pi)^{3} \delta^{(3)}\left(\boldsymbol{k}_{1}+\boldsymbol{k}_{2}+\boldsymbol{k}_{3}\right) \frac{\mathcal{C}_{5} H^{4}}{16 Q M_{\mathrm{pl}}^{2} c_{s}^{6}} \frac{1}{\left(k_{1} k_{2} k_{3}\right)^{3}}\left[\frac{k_{1}^{2}\left(\boldsymbol{k}_{2} \cdot \boldsymbol{k}_{3}\right)}{K}\left(1+\frac{k_{1}}{K}\right)+\mathrm{sym}\right], \\
& \left\langle\mathcal{R}\left(\boldsymbol{k}_{1}\right) \mathcal{R}\left(\boldsymbol{k}_{2}\right) \mathcal{R}\left(\boldsymbol{k}_{3}\right)\right\rangle^{(6)}=(2 \pi)^{3} \delta^{(3)}\left(\boldsymbol{k}_{1}+\boldsymbol{k}_{2}+\boldsymbol{k}_{3}\right) \frac{3 \mathcal{C}_{6} H^{6}}{4 Q^{3} c_{s}^{8}} \frac{1}{k_{1} k_{2} k_{3}} \frac{1}{K^{3}}, \\
& \left\langle\mathcal{R}\left(\boldsymbol{k}_{1}\right) \mathcal{R}\left(\boldsymbol{k}_{2}\right) \mathcal{R}\left(\boldsymbol{k}_{3}\right)\right\rangle^{(7)}=(2 \pi)^{3} \delta^{(3)}\left(\boldsymbol{k}_{1}+\boldsymbol{k}_{2}+\boldsymbol{k}_{3}\right) \frac{\mathcal{C}_{7} H^{6}}{8 Q^{3} c_{s}^{10}} \frac{1}{\left(k_{1} k_{2} k_{3}\right)^{3}} \frac{1}{K}\left[1+\frac{k_{1} k_{2}+k_{2} k_{3}+k_{3} k_{1}}{K^{2}}+\frac{3 k_{1} k_{2} k_{3}}{K^{3}}\right] \\
& \times\left[k_{1}^{2}\left(\boldsymbol{k}_{2} \cdot \boldsymbol{k}_{3}\right)-\left(\boldsymbol{k}_{1} \cdot \boldsymbol{k}_{2}\right)\left(\boldsymbol{k}_{1} \cdot \boldsymbol{k}_{3}\right)+\mathrm{sym}\right], \\
& \left\langle\mathcal{R}\left(\boldsymbol{k}_{1}\right) \mathcal{R}\left(\boldsymbol{k}_{2}\right) \mathcal{R}\left(\boldsymbol{k}_{3}\right)\right\rangle^{(8)}=(2 \pi)^{3} \delta^{(3)}\left(\boldsymbol{k}_{1}+\boldsymbol{k}_{2}+\boldsymbol{k}_{3}\right) \frac{\mathcal{C}_{8} H^{5}}{32 Q^{2} M_{\mathrm{pl}} c_{s}^{8}} \frac{1}{\left(k_{1} k_{2} k_{3}\right)^{3}} \frac{1}{K} \\
& \times\left\{\left(2+\frac{2 k_{1}+k_{2}+k_{3}}{K}+\frac{2 k_{1}\left(k_{2}+k_{3}\right)}{K^{2}}\right)\left[k_{1}^{2}\left(\boldsymbol{k}_{2} \cdot \boldsymbol{k}_{3}\right)-\left(\boldsymbol{k}_{1} \cdot \boldsymbol{k}_{2}\right)\left(\boldsymbol{k}_{1} \cdot \boldsymbol{k}_{3}\right)\right]+\mathrm{sym}\right\}
\end{aligned}
$$

where $K=k_{1}+k_{2}+k_{3}$. The symbol "sym" corresponds to the symmetric terms with respect to $k_{1}, k_{2}$, and $k_{3}$.

We express the three-point correlation function in the form

$$
\left\langle\mathcal{R}\left(\boldsymbol{k}_{1}\right) \mathcal{R}\left(\boldsymbol{k}_{2}\right) \mathcal{R}\left(\boldsymbol{k}_{3}\right)\right\rangle=(2 \pi)^{3} \delta^{(3)}\left(\boldsymbol{k}_{1}+\boldsymbol{k}_{2}+\boldsymbol{k}_{3}\right)\left(\mathcal{P}_{\mathcal{R}}\right)^{2} B_{\mathcal{R}}\left(k_{1}, k_{2}, k_{3}\right),
$$

where $\mathcal{P}_{\mathcal{R}}$ is given by Eq. (31), and

$$
B_{\mathcal{R}}\left(k_{1}, k_{2}, k_{3}\right)=\frac{(2 \pi)^{4}}{\prod_{i=1}^{3} k_{i}^{3}} \mathcal{A}_{\mathcal{R}} .
$$


Collecting all the terms in Eqs. (75)-(82) we have

$$
\begin{aligned}
\mathcal{A}_{\mathcal{R}}=\frac{M_{\mathrm{pl}}^{2}}{Q}\left\{\frac{1}{4}\left(\frac{2}{K} \sum_{i>j} k_{i}^{2} k_{j}^{2}-\frac{1}{K^{2}} \sum_{i \neq j} k_{i}^{2} k_{j}^{3}\right) \mathcal{C}_{1}+\frac{1}{4 c_{s}^{2}}\left(\frac{1}{2} \sum_{i} k_{i}^{3}+\frac{2}{K} \sum_{i>j} k_{i}^{2} k_{j}^{2}-\frac{1}{K^{2}} \sum_{i \neq j} k_{i}^{2} k_{j}^{3}\right) \mathcal{C}_{2}\right. \\
+\frac{3}{2} \frac{H}{M_{\mathrm{pl}}} \frac{\left(k_{1} k_{2} k_{3}\right)^{2}}{K^{3}} \mathcal{C}_{3}+\frac{1}{8} \frac{Q}{M_{\mathrm{pl}}^{2}}\left(\sum_{i} k_{i}^{3}-\frac{1}{2} \sum_{i \neq j} k_{i} k_{j}^{2}-\frac{2}{K^{2}} \sum_{i \neq j} k_{i}^{2} k_{j}^{3}\right) \mathcal{C}_{4} \\
+\frac{1}{4}\left(\frac{Q}{M_{\mathrm{pl}}^{2}}\right)^{2} \frac{1}{K^{2}}\left[\sum_{i} k_{i}^{5}+\frac{1}{2} \sum_{i \neq j} k_{i} k_{j}^{4}-\frac{3}{2} \sum_{i \neq j} k_{i}^{2} k_{j}^{3}-k_{1} k_{2} k_{3} \sum_{i>j} k_{i} k_{j}\right] \mathcal{C}_{5}+\frac{3}{c_{s}^{2}}\left(\frac{H}{M_{\mathrm{pl}}}\right)^{2} \frac{\left(k_{1} k_{2} k_{3}\right)^{2}}{K^{3}} \mathcal{C}_{6} \\
+\frac{1}{2 c_{s}^{4}}\left(\frac{H}{M_{\mathrm{pl}}}\right)^{2} \frac{1}{K}\left(1+\frac{1}{K^{2}} \sum_{i>j} k_{i} k_{j}+\frac{3 k_{1} k_{2} k_{3}}{K^{3}}\right)\left[\frac{3}{4} \sum_{i} k_{i}^{4}-\frac{3}{2} \sum_{i>j} k_{i}^{2} k_{j}^{2}\right] \mathcal{C}_{7} \\
\left.+\frac{1}{8 c_{s}^{2}} \frac{H}{M_{\mathrm{pl}}} \frac{Q}{M_{\mathrm{pl}}^{2}} \frac{1}{K^{2}}\left[\frac{3}{2} k_{1} k_{2} k_{3} \sum_{i} k_{i}^{2}-\frac{5}{2} k_{1} k_{2} k_{3} K^{2}-6 \sum_{i \neq j} k_{i}^{2} k_{j}^{3}-\sum_{i} k_{i}^{5}+\frac{7}{2} K \sum_{i} k_{i}^{4}\right] \mathcal{C}_{8}\right\} .
\end{aligned}
$$

We also define the non-linear parameter $f_{\mathrm{NL}}$, as [2, 58]

$$
f_{\mathrm{NL}}=\frac{10}{3} \frac{\mathcal{A}_{\mathcal{R}}}{\sum_{i=1}^{3} k_{i}^{3}} .
$$

If the three-point correlation function is described by the equilateral configuration $\left(k_{1}=k_{2}=k_{3}\right)$, it follows that

$$
\begin{aligned}
f_{\mathrm{NL}}=\frac{40}{9} \frac{M_{\mathrm{pl}}^{2}}{Q} & {\left[\frac{1}{12} \mathcal{C}_{1}+\frac{17}{96 c_{s}^{2}} \mathcal{C}_{2}+\frac{1}{72} \frac{H}{M_{\mathrm{pl}}} \mathcal{C}_{3}-\frac{1}{24} \frac{Q}{M_{\mathrm{pl}}^{2}} \mathcal{C}_{4}-\frac{1}{24}\left(\frac{Q}{M_{\mathrm{pl}}^{2}}\right)^{2} \mathcal{C}_{5}+\frac{1}{36 c_{s}^{2}}\left(\frac{H}{M_{\mathrm{pl}}}\right)^{2} \mathcal{C}_{6}\right.} \\
& \left.-\frac{13}{96 c_{s}^{4}}\left(\frac{H}{M_{\mathrm{pl}}}\right)^{2} \mathcal{C}_{7}-\frac{17}{192 c_{s}^{2}} \frac{H}{M_{\mathrm{pl}}} \frac{Q}{M_{\mathrm{pl}}^{2}} \mathcal{C}_{8}\right] .
\end{aligned}
$$

This is the same form as that derived in Ref. [45], but the coefficients $\mathcal{C}_{i}(i=1, \cdots, 8)$ are different.

\section{THE SHAPES OF NON-GAUSSIANITIES}

The shapes of non-Gaussianities have been discussed by a number of authors (see e.g., Refs. [24, 59]). In Ref. [24] Chen et al. studied the case of k-inflation, where only the terms proportional to $\mathcal{C}_{i}$ (with $1 \leq i \leq 5$ ) are present. Here we wish to discuss the shapes coming from the remaining terms, that is, we focus our attention only on the following terms

$$
\begin{aligned}
\mathcal{B}_{\mathcal{R}}^{(6)} & =\frac{1}{\sum_{i=1}^{3} k_{i}^{3}} \frac{3 H^{2}}{Q c_{s}^{2}} \frac{\left(k_{1} k_{2} k_{3}\right)^{2}}{K^{3}} \mathcal{C}_{6}, \\
\mathcal{B}_{\mathcal{R}}^{(7)} & =\frac{1}{\sum_{i=1}^{3} k_{i}^{3}} \frac{H^{2}}{2 Q c_{s}^{4}} \frac{1}{K}\left(1+\frac{1}{K^{2}} \sum_{i>j} k_{i} k_{j}+\frac{3 k_{1} k_{2} k_{3}}{K^{3}}\right)\left[\frac{3}{4} \sum_{i} k_{i}^{4}-\frac{3}{2} \sum_{i>j} k_{i}^{2} k_{j}^{2}\right] \mathcal{C}_{7}, \\
\mathcal{B}_{\mathcal{R}}^{(8)} & =\frac{1}{\sum_{i=1}^{3} k_{i}^{3}} \frac{1}{8 c_{s}^{2}} \frac{H}{M_{\mathrm{pl}}} \frac{1}{K^{2}}\left[\frac{3}{2} k_{1} k_{2} k_{3} \sum_{i} k_{i}^{2}-\frac{5}{2} k_{1} k_{2} k_{3} K^{2}-6 \sum_{i \neq j} k_{i}^{2} k_{j}^{3}-\sum_{i} k_{i}^{5}+\frac{7}{2} K \sum_{i} k_{i}^{4}\right] \mathcal{C}_{8} .
\end{aligned}
$$

Although the total bispectrum is the sum of all the previous contributions (in addition to the standard ones of k-inflation), we study the momentum dependence of each single contribution $\mathcal{B}_{\mathcal{R}}^{(i)}$ (with $6 \leq i \leq 8$ ). In fact, we will check that the non-Gaussianities associated to each single term $\mathcal{B}_{\mathcal{R}}^{(i)}$ can be well measured by means of the equilateral estimator defined as

$$
\mathcal{B}_{\mathcal{R}}^{\text {equil }} \equiv(2 \pi)^{4}\left(\frac{9}{10} f_{\mathrm{NL}}^{\text {equil }}\right)\left[-\frac{1}{k_{1}^{3} k_{2}^{3}}-\frac{1}{k_{1}^{3} k_{3}^{3}}-\frac{1}{k_{2}^{3} k_{3}^{3}}-\frac{2}{k_{1}^{2} k_{2}^{2} k_{3}^{2}}+\frac{1}{k_{1} k_{2}^{2} k_{3}^{3}}+(5 \text { perms. })\right] .
$$



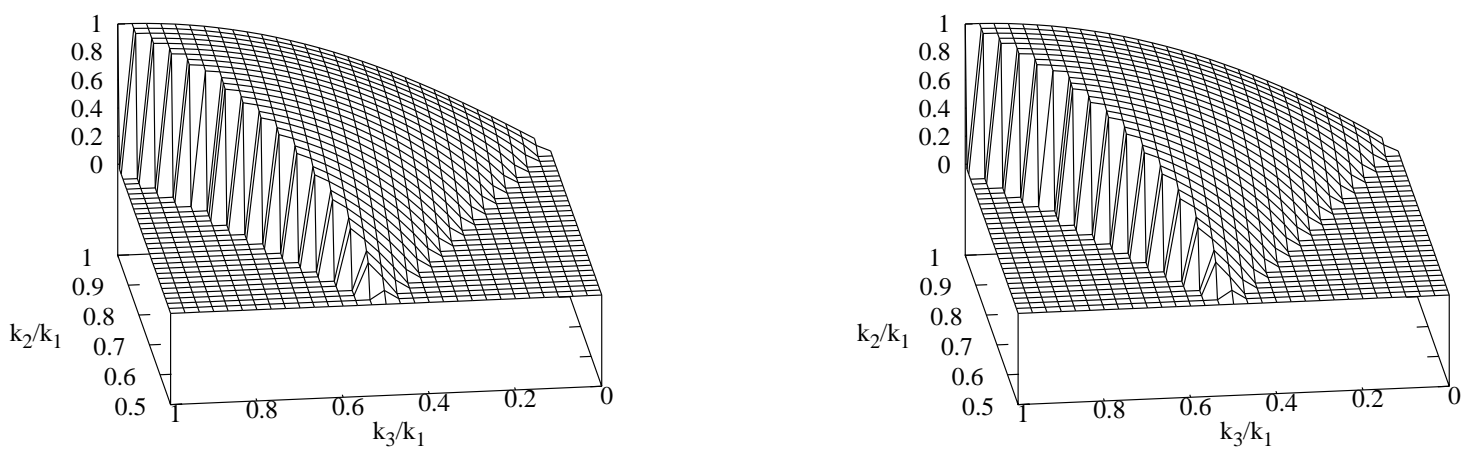

Figure 1: The shape functions $\mathcal{B}_{\mathcal{R}}^{(7)}\left(1, k_{2} / k_{1}, k_{3} / k_{1}\right)\left(k_{2} / k_{1}\right)^{2}\left(k_{3} / k_{1}\right)^{2}$ (left) and $\mathcal{B}_{\mathcal{R}}^{(8)}\left(1, k_{2} / k_{1}, k_{3} / k_{1}\right)\left(k_{2} / k_{1}\right)^{2}\left(k_{3} / k_{1}\right)^{2}($ right $)$. The two functions are plotted in the domain $1-k_{2} / k_{1} \leq k_{3} / k_{1} \leq k_{2} / k_{1}$. The lower boundary is given by the triangular inequality, whereas the upper boundary is chosen in order not to repeat twice the same physical configuration. Finally, the functions $\mathcal{B}_{\mathcal{R}}^{(i)}$ are multiplied by the measure $\left(k_{2} / k_{1}\right)^{2}\left(k_{3} / k_{1}\right)^{2}$ following Ref. [59]. The plots are normalized to have a unit value at the point $k_{2} / k_{1}=k_{3} / k_{1}=1$.

In Ref. [44] it was shown that $\mathcal{B}_{\mathcal{R}}^{(6)}$ exhibits the same momentum dependence as that of the term $\mathcal{B}_{\mathcal{R}}^{(3)}$, which has a high correlation with $\mathcal{B}_{\mathcal{R}}^{\text {equil }}$ (about 0.936 ). Therefore, we further restrict our analysis only to the new non-trivial terms $\mathcal{B}_{\mathcal{R}}^{(7)}$ and $\mathcal{B}_{\mathcal{R}}^{(8)}$. In Fig. 1 we plot the shapes of the bispectrum contributions $\mathcal{B}_{\mathcal{R}}^{(7)}$ and $\mathcal{B}_{\mathcal{R}}^{(8)}$.

Let us quantify how much the shape functions of $\mathcal{B}_{\mathcal{R}}^{(i)}$ can be fitted with the function $\mathcal{B}_{\mathcal{R}}^{\text {equil }}$. We follow the procedure shown in Ref. [60] and calculate the shape correlator $C\left(\mathcal{B}_{\mathcal{R}}, \mathcal{B}_{\mathcal{R}}^{\prime}\right)$ defined as

$$
C\left(\mathcal{B}_{\mathcal{R}}, \mathcal{B}_{\mathcal{R}}^{\prime}\right)=\frac{\mathcal{I}\left(\mathcal{B}_{\mathcal{R}}, \mathcal{B}_{\mathcal{R}}^{\prime}\right)}{\sqrt{\mathcal{I}\left(\mathcal{B}_{\mathcal{R}}, \mathcal{B}_{\mathcal{R}}\right) \mathcal{I}\left(\mathcal{B}_{\mathcal{R}}^{\prime}, \mathcal{B}_{\mathcal{R}}^{\prime}\right)}}
$$

where

$$
\mathcal{I}\left(\mathcal{B}_{\mathcal{R}}, \mathcal{B}_{\mathcal{R}}^{\prime}\right)=\int d \mathcal{V}_{k} \mathcal{B}_{\mathcal{R}}\left(k_{1}, k_{2}, k_{3}\right) \mathcal{B}_{\mathcal{R}}^{\prime}\left(k_{1}, k_{2}, k_{3}\right) \frac{k_{1}^{4} k_{2}^{4} k_{3}^{4}}{\left(k_{1}+k_{2}+k_{3}\right)^{3}}
$$

and the region of integration is defined by the condition $0 \leq k_{1}<\infty, 0<k_{2} / k_{1}<1$, and $1-k_{2} / k_{1} \leq k_{3} / k_{1} \leq 1$. In terms of the variables $k_{2} / k_{1}$ and $k_{3} / k_{1}$ the integral in $k_{1}$ factorizes out. After performing numerical integrations we find that

$$
C\left(\mathcal{B}_{\mathcal{R}}^{(7)}, \mathcal{B}_{\mathcal{R}}^{\text {equil }}\right)=-0.99989 \quad \text { and } \quad C\left(\mathcal{B}_{\mathcal{R}}^{(8)}, \mathcal{B}_{\mathcal{R}}^{\text {equil }}\right)=-0.99999
$$

Since both the contributions $\mathcal{B}_{\mathcal{R}}^{(7)}$ and $\mathcal{B}_{\mathcal{R}}^{(8)}$ are almost completely anti-correlated with $\mathcal{B}_{\mathcal{R}}^{\text {equil }}$, it makes sense to use $f_{\mathrm{NL}}^{\text {equil }}$ to measure the shapes of non-Gaussianities in the presence of the contributions $\mathcal{B}_{\mathcal{R}}^{(7)}$ and $\mathcal{B}_{\mathcal{R}}^{(8)}$ as well. We also checked that $C\left(\mathcal{B}_{\mathcal{R}}^{(6)}, \mathcal{B}_{\mathcal{R}}^{\text {equil }}\right)=0.936177$, which agrees with the result in Ref. [44]. 


\section{THE NON-LINEAR PARAMETER UNDER THE SLOW-VARIATION APPROXIMATION}

In this section we derive the approximate expression of the equilateral non-linear parameter $f_{\mathrm{NL}}^{\text {equil }}$ with $k_{1}=k_{2}=k_{3}$ on the quasi de Sitter background. In order to obtain a compact form of $f_{\mathrm{NL}}^{\text {equil }}$ we define the following quantities

$$
\begin{aligned}
\Sigma \equiv & \frac{w_{1}\left(4 w_{1} w_{3}+9 w_{2}^{2}\right)}{12 M_{\mathrm{pl}}^{4}}, \\
\lambda \equiv & \frac{F^{2}}{3}\left[3 X^{2} P_{, X X}+2 X^{3} P_{, X X X}+3 H \dot{\phi}\left(X G_{3, X}+5 X^{2} G_{3, X X}+2 X^{3} G_{3, X X X}\right)-2\left(2 X^{2} G_{3, \phi X}+X^{3} G_{3, \phi X X}\right)\right. \\
& +6 H^{2}\left(9 X^{2} G_{4, X X}+16 X^{3} G_{4, X X X}+4 X^{4} G_{4, X X X X}\right)-3 H \dot{\phi}\left(3 X G_{4 \phi, X}+12 X^{2} G_{4, \phi X X}+4 X^{3} G_{4, \phi X X X}\right) \\
& +H^{3} \dot{\phi}\left(3 X G_{5, X}+27 X^{2} G_{5, X X}+24 X^{3} G_{5, X X X}+4 X^{4} G_{5, X X X X}\right) \\
& \left.-6 H^{2}\left(6 X^{2} G_{5, \phi X}+9 X^{3} G_{5, \phi X X}+2 X^{4} G_{5, \phi X X X}\right)\right],
\end{aligned}
$$

which are the generalizations of those introduced in Refs. [22, 44, 45]. Notice that $\Sigma$ is related with $Q$ via $Q=$ $4 M_{\mathrm{pl}}^{4} \Sigma / w_{2}^{2}$. We also introduce the following quantities

$$
\lambda_{3 X} \equiv \frac{X G_{3, X X}}{G_{3, X}}, \quad \lambda_{4 X} \equiv \frac{X G_{4, X X X}}{G_{4, X X}}, \quad \lambda_{5 X} \equiv \frac{X G_{5, X X X}}{G_{5, X X}} .
$$

We derive the leading-order terms to each $f_{\mathrm{NL}}^{\text {equil }(i)}$ coming from the coefficients $\mathcal{C}_{i}(i=1, \cdots, 8)$. We expand each $f_{\mathrm{NL}}^{\text {equil }(i)}$ in terms of the slow-variation parameters defined in Eq. (10), by treating $c_{s}^{2}, \lambda / \Sigma$, and $\lambda_{i X}(i=3,4,5)$ as arbitrary parameters. Then the leading-order contributions to $f_{\mathrm{NL}}^{\text {equil }(i)}$ are given by

$$
\begin{aligned}
f_{\mathrm{NL}}^{\text {equil (1) }=} & \frac{10}{9}\left(1-\frac{1}{c_{s}^{2}}\right)+\frac{10}{27 c_{s}^{2}}\left(\epsilon_{s}-\eta_{s}-4 \delta_{G 3 X}-12 \delta_{G 4 X}-32 \delta_{G 4 X X}+12 \delta_{G 5 \phi}-10 \delta_{G 5 X}-8 \delta_{G 5 X X}\right) \\
f_{\mathrm{NL}}^{\text {equil }(2)}= & \frac{85}{108}\left(\frac{1}{c_{s}^{2}}-1\right)+\frac{85}{108 c_{s}^{2}}\left(\epsilon_{s}+\eta_{s}-2 s+4 \delta_{G 4 X}+2 \delta_{G 5 X}-4 \delta_{G 5 \phi}\right) \\
f_{\mathrm{NL}}^{\text {equil (3) }=} & \frac{5}{81}\left(\frac{1}{c_{s}^{2}}-1\right)-\frac{10}{81} \frac{\lambda}{\Sigma}+\frac{5}{81 c_{s}^{2}}\left(\delta_{G 3 X}+4 \delta_{G 4 X}+3 \delta_{G 5 X}-\delta_{G 4 \phi}-4 \delta_{G 5 \phi}+8 \delta_{G 4 X X}+2 \delta_{G 5 X X}\right) \\
& -\frac{5}{81}\left(3+2 \lambda_{3 X}\right) \delta_{G 3 X}-\frac{40}{81}\left(5+2 \lambda_{4 X}\right) \delta_{G 4 X X}-\frac{20}{81}\left(4+\lambda_{5 X}\right) \delta_{G 5 X X} \\
& +\frac{5}{81}\left(\delta_{G 4 \phi}+8 \delta_{G 5 \phi}-8 \delta_{G 4 X}-9 \delta_{G 5 X}\right)-\frac{10}{27} \frac{c_{s}^{2}}{\epsilon_{s}}\left[\left(1+\lambda_{3 X}\right) \delta_{G 3 X}^{2}+\xi\left(\delta^{2}\right)\right] \\
f_{\mathrm{NL}}^{\text {equil (4) }=} & \frac{10}{27} \frac{\epsilon_{s}}{c_{s}^{2}}, \\
f_{\mathrm{NL}}^{\text {equil (5) }=} & -\frac{5}{108 c_{s}^{2}}\left(\epsilon_{s}-4 \delta_{G 3 X}-8 \delta_{G 4 X X}+8 \delta_{G 5 X}+4 \delta_{G 5 X X}\right) \epsilon_{s}, \\
f_{\mathrm{NL}}^{\text {equil (6) }=} & \frac{20}{81 \epsilon_{s}}\left[\left(1+\lambda_{3 X}\right) \delta_{G 3 X}+4\left(3+2 \lambda_{4 X}\right) \delta_{G 4 X X}+\delta_{G 5 X}+\left(5+2 \lambda_{5 X}\right) \delta_{G 5 X X}\right] \\
f_{\mathrm{NL}}^{\text {equil (7) }=} & \frac{65}{162 c_{s}^{2} \epsilon_{s}}\left(\delta_{G 3 X}+6 \delta_{G 4 X X}+\delta_{G 5 X}+\delta_{G 5 X X}\right) \\
f_{\mathrm{NL}}^{\text {equil (8) }=} & -\frac{85}{108 c_{s}^{2}}\left(\delta_{G 3 X}+4 \delta_{G 4 X X}\right) .
\end{aligned}
$$

The explicit expression of the second-order term $\xi\left(\delta^{2}\right)$ in $f_{\mathrm{NL}}^{\text {equil (3) }}$ is given in Appendix. The above results reproduce those obtained for the theories with $G_{4}=0$ and $G_{5}=0$ [45].

Summing up all the terms $f_{\mathrm{NL}}^{\text {equil }(i)}(i=1, \cdots, 8)$ and taking the largest contribution, we obtain

$$
\begin{aligned}
f_{\mathrm{NL}}^{\text {equil }}= & \frac{85}{324}\left(1-\frac{1}{c_{s}^{2}}\right)-\frac{10}{81} \frac{\lambda}{\Sigma}+\frac{55}{36} \frac{\epsilon_{s}}{c_{s}^{2}}+\frac{5}{12} \frac{\eta_{s}}{c_{s}^{2}}-\frac{85}{54} \frac{s}{c_{s}^{2}}+\left(\frac{20}{81} \frac{1+\lambda_{3 X}}{\epsilon_{s}}+\frac{65}{162 c_{s}^{2} \epsilon_{s}}\right) \delta_{G 3 X} \\
& +\left(\frac{80}{81} \frac{3+2 \lambda_{4 X}}{\epsilon_{s}}+\frac{65}{27 c_{s}^{2} \epsilon_{s}}\right) \delta_{G 4 X X}+\left(\frac{20}{81 \epsilon_{s}}+\frac{65}{162 c_{s}^{2} \epsilon_{s}}\right) \delta_{G 5 X}+\left(\frac{20}{81} \frac{5+2 \lambda_{5 X}}{\epsilon_{s}}+\frac{65}{162 c_{s}^{2} \epsilon_{s}}\right) \delta_{G 5 X X}
\end{aligned}
$$


Here we have ignored the contributions such as $\delta_{G 4 X}, \delta_{G 4 \phi}$ and $\delta_{G 5 \phi}$ relative to the terms $\delta_{G 4 X X} / \epsilon_{s}, \delta_{G 5 X} / \epsilon_{s}$, and $\delta_{G 5 X X} / \epsilon_{s}$.

From Eq. (106) we find that the scalar propagation speed $c_{s}$ mainly determines the level of non-Gaussianities. Expanding the term $Q$ in Eq. (26) in terms of slow-variation parameters, the leading-order contribution is

$$
\begin{gathered}
Q \simeq M_{\mathrm{pl}}^{2} F\left[\delta_{P X}\left(1+2 \lambda_{P X}\right)+6 \delta_{G 3 X}\left(1+\lambda_{3 X}\right)-2 \delta_{3 \phi}+6 \delta_{G 4 X}+24 \delta_{G 4 X X}\left(2+\lambda_{4 X}\right)\right. \\
\left.+6 \delta_{G 5 X}+2 \delta_{G 5 X X}\left(7+2 \lambda_{5 X}\right)-6 \delta_{G 5 \phi}\right]
\end{gathered}
$$

where $\lambda_{i X}(i=3,4,5)$ are defined in Eq. (97) and

$$
\lambda_{P X}=\frac{X P_{, X X}}{P_{, X}}
$$

Then the scalar propagation speed squared $c_{s}^{2}=M_{\mathrm{pl}}^{2} F \epsilon_{s} / Q$ is approximately given by

$$
\begin{aligned}
c_{s}^{2} \simeq & \left(\delta_{P X}+4 \delta_{G 3 X}-2 \delta_{G 3 \phi}+6 \delta_{G 4 X}+20 \delta_{G 4 X X}+4 \delta_{G 5 X}+4 \delta_{G 5 X X}-6 \delta_{G 5 \phi}\right) /\left[\delta_{P X}\left(1+2 \lambda_{P X}\right)+6 \delta_{G 3 X}\left(1+\lambda_{3 X}\right)\right. \\
& \left.-2 \delta_{G 3 \phi}+6 \delta_{G 4 X}+24 \delta_{G 4 X X}\left(2+\lambda_{4 X}\right)+6 \delta_{G 5 X}+2 \delta_{G 5 X X}\left(7+2 \lambda_{5 X}\right)-6 \delta_{G 5 \phi}\right],
\end{aligned}
$$

where we have used Eq. (30). One can estimate the ratio $\lambda / \Sigma$ in Eq. (106) as follows

$$
\begin{aligned}
\frac{\lambda}{\Sigma} \simeq & {\left[\delta_{P X}\left(3 \lambda_{P X}+2 \lambda_{P X X}\right)+3 \delta_{G 3 X}\left(1+5 \lambda_{3 X}+2 \lambda_{3 X X}\right)+6 \delta_{G 4 X X}\left(9+16 \lambda_{4 X}+4 \lambda_{4 X X}\right)+3 \delta_{G 5 X}\right.} \\
& \left.+\delta_{G 5 X X}\left(27+24 \lambda_{5 X}+4 \lambda_{5 X X}\right)\right] / 3\left[\delta_{P X}\left(1+2 \lambda_{P X}\right)+6 \delta_{G 3 X}\left(1+\lambda_{3 X}\right)-2 \delta_{G 3 \phi}+6 \delta_{G 4 X}\right. \\
& \left.+24 \delta_{G 4 X X}\left(2+\lambda_{4 X}\right)+6 \delta_{G 5 X}-6 \delta_{G 5 \phi}+2 \delta_{G 5 X X}\left(7+2 \lambda_{5 X}\right)\right]
\end{aligned}
$$

where

$$
\lambda_{P X X}=\frac{X^{2} P_{, X X X}}{P_{, X}}, \quad \lambda_{3 X X}=\frac{X^{2} G_{3, X X X}}{G_{3, X}}, \quad \lambda_{4 X X}=\frac{X^{2} G_{4, X X X X}}{G_{4, X X}}, \quad \lambda_{5 X X}=\frac{X^{2} G_{5, X X X X}}{G_{5, X X}} .
$$

Let us consider the case in which either of the following conditions is satisfied:

$$
\lambda_{P X} \gg 1, \quad \lambda_{3 X} \gg 1, \quad \lambda_{4 X} \gg 1, \quad \lambda_{5 X} \gg 1 .
$$

Then one can realize $c_{s}^{2} \ll 1$ and hence $\left|f_{\mathrm{NL}}^{\text {equil }}\right| \gg 1$. From Eq. (110) it is also possible to have $\lambda / \Sigma \gg 1$ if either of $\lambda_{P X X}, \lambda_{3 X X}, \lambda_{4 X X}, \lambda_{5 X X}$ is much larger than 1 .

\section{APPLICATIONS TO CONCRETE MODELS OF INFLATION}

In this section we apply our formula for the equilateral non-linear parameter $f_{\mathrm{NL}}^{\text {equil }}$ to concrete models of inflationsuch as (A) k-inflation, (B) k-inflation in the presence of the terms $G_{i}(X)(i=3,4,5),(\mathrm{C})$ potential-driven Galileon inflation, (D) non-minimal coupling models, and (E) potential-driven inflation in the presence of the Gauss-Bonnet term.

\section{A. k-inflation}

In k-inflation in which $G_{3}=G_{4}=G_{5}=0$ one has $c_{s}^{2}=1 /\left(1+2 \lambda_{P X}\right)$ and $\lambda / \Sigma=\left(1-c_{s}^{2}\right) / 2+2 \lambda_{P X X} c_{s}^{2} / 3$. From Eq. (106) it follows that

$$
f_{\mathrm{NL}}^{\mathrm{equil}} \simeq \frac{5}{324}\left(1-\frac{1}{c_{s}^{2}}\right)\left(17+4 c_{s}^{2}\right)-\frac{20}{243} \frac{\lambda_{P X X}}{1+2 \lambda_{P X}}+\frac{55}{36} \frac{\epsilon_{s}}{c_{s}^{2}}+\frac{5}{12} \frac{\eta_{s}}{c_{s}^{2}}-\frac{85}{54} \frac{s}{c_{s}^{2}}
$$

Since $\epsilon=\delta_{P X}=P_{, X} X /\left(3 M_{\mathrm{pl}}^{2} H^{2}\right)[62,63]$, inflation occurs either around $P_{, X} \approx 0$ or $X \approx 0$.

In the former case $\left(P_{, X} \approx 0\right)$, as long as $P_{, X X}$ does not vanish at $P_{, X}=0$, we have $\lambda_{P X}=X P_{, X X} / P_{, X} \gg 1$ and $c_{s}^{2} \ll 1$. Hence large non-Gaussianities can be realized for the Lagrangian having a non-linear term in $X$. The ghost condensate model $P=-X+X^{2} /\left(2 M^{4}\right)[18]$ belongs to this class. For the function $P$ depending only on $X$ there is a de Sitter solution at $P_{, X}=0$. However this is problematic because the scalar power spectrum $\mathcal{P}_{\mathcal{R}}$ diverges at the de 
Sitter solution (because $c_{s}=0$ ) [15]. This problem can be avoided either by involving the $\phi$-dependence in $P$ (such as the dilatonic ghost condensate model $\left.P=-X+e^{\lambda \phi / M_{\mathrm{pl}}} X^{2} /\left(2 M^{4}\right)[19]\right)$ or by taking into account the terms $G_{i}(X)$ $(i=3,4,5)$ in addition to the Lagrangian $P(X)[20$. We shall discuss the latter case in Sec. VIIB]

If inflation occurs in the region $X \approx 0$, whether large non-Gaussianities can be realized or not depends on the models. In conventional inflation driven by the potential energy $V(\phi)$ of the field $\phi$, i.e. $P=X-V(\phi)$, we have $c_{s}^{2}=1$ and $\lambda_{P X X}=0$, which leads to the small non-linear parameter $f_{\mathrm{NL}}^{\text {equil }}=55 \epsilon_{s} / 36+5 \eta_{s} / 12$. In the DBI model where the Lagrangian is given by $P=-\sqrt{1-2 f(\phi) X} / f(\phi)+1 / f(\phi)-V(\phi)$ [16], it follows that $\lambda_{P X}=f(\phi) X /[1-2 f(\phi) X]$ and $\epsilon=X /\left[M_{\mathrm{pl}}^{2} H^{2} \sqrt{1-2 f(\phi) X}\right]$. The non-Gaussianities can be large around the region $2 f(\phi) X \approx 1$. If the total energy density is dominated by the potential energy $V$, i.e. $M_{\mathrm{pl}}^{2} H^{2} \approx V / 3$, it is possible to satisfy the condition $\epsilon \ll 1$ for $X \ll V$ (even if $2 f(\phi) X$ is close to 1 ). In fact this corresponds to the regime where the non-linear parameter $f_{\mathrm{NL}}^{\text {equil }}$ of the order of 10 can be achieved [17].

\section{B. k-inflation with the terms $G_{i}(X)(i=3,4,5)$}

In k-inflation models where the Lagrangian $P$ is a function of $X$ only, the problem of the de Sitter solution mentioned in Sec. VIIA can be circumvented by taking into account the terms $G_{i}(X)(i=3,4,5)$. For the ghost condensate model $P=-X+X^{2} /\left(2 M^{4}\right)$ with the term $G_{3}=\mu X / M^{4}(\mu>0, M>0)$ [20], for example, the scalar power spectrum is not divergent because $c_{s}^{2} \neq 0$. In this case, in the region where the variable $x=X / M^{4}$ is close to 1 , we find that $1-x \simeq \sqrt{3} \mu / M_{\mathrm{pl}}$ and $f_{\mathrm{NL}}^{\text {equil }} \simeq 5 /[6(1-x)] \simeq 4.62 r^{-2 / 3}$, where $r \simeq 16 \sqrt{6}(1-x)^{3 / 2} / 3$. These results match with those found in Ref. [44] (in which detailed calculations are given).

Let us consider the following model

$$
P=-X+\frac{X^{2}}{2 M^{4}}, \quad G_{4}=\mu \frac{X^{2}}{M^{7}}
$$

where $\mu$ and $M$ are positive constants. There is a de Sitter solution characterized by the condition $\epsilon=\delta_{P X}+6 \delta_{G 4 X}+$ $12 \delta_{G 4 X X}=0$, at which $P+3 M_{\mathrm{pl}}^{2} H^{2} F-12 H^{2} X G_{4, X}=0$ from Eq. (5). We then obtain

$$
H^{2}=\frac{M^{3}}{36 \mu} \frac{1-x}{x}, \quad \frac{\mu M}{M_{\mathrm{pl}}^{2}}=\frac{1-x}{6 x^{2}(3-2 x)},
$$

where $x=X / M^{4}(>0)$. Provided that inflation occurs in the regime $\mu M \ll M_{\mathrm{pl}}^{2}$, the variable $x$ is close to 1 (with $x<1$ ). In the following we replace $x$ for 1 apart from the terms including $1-x$. From Eq. (115) we have $\mu M / M_{\mathrm{pl}}^{2} \simeq(1-x) / 6$ and $H^{2} \simeq M^{4} /\left(6 M_{\mathrm{pl}}^{2}\right)$. Since $Q \simeq 12 M_{\mathrm{pl}}^{2}>0$, the no-ghost condition is satisfied. The scalar propagation speed squared is given by $c_{s}^{2} \simeq 2(1-x) / 9$, so that the Laplacian instability is absent for $x<1$. Since $\epsilon_{s} \simeq 8(1-x) / 3$, the scalar power spectrum and the tensor-to-scalar ratio are given by

$$
\mathcal{P}_{\mathcal{R}} \simeq \frac{3 \sqrt{2}}{256 \pi^{2}}\left(\frac{M}{M_{\mathrm{pl}}}\right)^{4} \frac{1}{(1-x)^{3 / 2}}, \quad r \simeq \frac{128 \sqrt{2}}{9}(1-x)^{3 / 2} .
$$

The term $\lambda / \Sigma$ in Eq. (110) is estimated to be $\lambda / \Sigma \simeq 1 / 2$. Since $\delta_{G 4 X X} \simeq(1-x) / 3$, we find that the equilateral non-linear parameter is given by

$$
f_{\mathrm{NL}}^{\mathrm{equil}} \simeq \frac{25}{144} \frac{1}{1-x} \simeq 1.28 r^{-2 / 3}
$$

For smaller $r, f_{\mathrm{NL}}^{\text {equil }}$ gets larger. If $r=0.01$, then $f_{\mathrm{NL}}^{\text {equil }}=9.4$. This level of non-Gaussianities can be detectable in future observations.

We also consider the following model

$$
P=-X+\frac{X^{2}}{2 M^{4}}, \quad G_{5}=\mu \frac{X^{2}}{M^{10}} .
$$

A similar calculation shows that there is a de Sitter solution characterized by $\mu^{2} M^{4} / M_{\mathrm{pl}}^{6} \simeq 27(1-x)^{2} / 25$ and $H^{2} \simeq M^{4} /\left(6 M_{\mathrm{pl}}^{2}\right)$ for $x=X / M^{4}$ close to 1 . Since $\epsilon_{s} \simeq 18(1-x) / 5, c_{s}^{2} \simeq 3(1-x) / 10, \delta_{G 5 X}=\delta_{G 5 X X} \simeq 6(1-x) / 5$, $\lambda / \Sigma \simeq 1 / 2$, and $Q \simeq 12 M_{\mathrm{pl}}^{2}>0$ in this case, it follows that

$$
f_{\mathrm{NL}}^{\text {equil }} \simeq \frac{25}{1458} \frac{1}{1-x} \simeq 0.17 r^{-2 / 3}
$$

which is about one order of magnitude smaller than (117). 


\section{Potential-driven Galileon inflation}

Let us proceed to the potential-driven inflation $[P=X-V(\phi)]$ in the presence of the Galileon term $G_{3}(X) \propto X^{n}$ $(n>0)$ [38]. The inflationary dynamics for the case $n=1$ were studied in Refs. [33, 38]. Since $\lambda_{P X}=0$ and $\lambda_{3 X}=n-1$ we have

$$
c_{s}^{2}=\frac{\delta_{P X}+4 \delta_{G 3 X}}{\delta_{P X}+6 n \delta_{G 3 X}} .
$$

In the regime $\delta_{G 3 X} \gg \delta_{P X}$ it follows that $c_{s}^{2} \simeq 2 /(3 n)$ and hence $c_{s}^{2} \ll 1$ for $n \gg 1$. In this case one has $\epsilon_{s} \simeq 4 \delta_{G 3 X}$ and $\lambda / \Sigma \simeq n / 3$, so that the non-linear parameter (106) is given by

$$
f_{\mathrm{NL}}^{\mathrm{equil}} \simeq-\frac{865}{3888} n
$$

In the presence of the term $G_{4}(X) \propto X^{n}$ the slow-variation parameter $\delta_{G 4 X X}$ is related with $\delta_{G 4 X}$, via $\delta_{G 4 X X}=$ $(n-1) \delta_{G 4 X}$. If $n \gg 1$, one has $c_{s}^{2} \simeq 5 /(6 n), \lambda / \Sigma \simeq n / 3, \epsilon_{s} \simeq 20 \delta_{G 4 X X}$ in the regime $1 \gg \delta_{G 4 X X} \gg \delta_{P X}$, and hence $f_{\mathrm{NL}}^{\text {equil }} \simeq-137 n / 1215$. Similarly, in the case where $G_{5}(X) \propto X^{n}$ with $n \gg 1$, it follows that $f_{\mathrm{NL}}^{\text {equil }} \simeq-155 n / 1944$ for $1 \gg \delta_{G 5 X X} \gg \delta_{P X}$.

\section{Nonminimal coupling models}

The nonminimal coupling of the scalar field $\phi$ with the Ricci scalar $R$ corresponds to the choice $G_{4}=F(\phi)$, where $F(\phi)$ is an arbitrary function in terms of $\phi$. In the absence of the terms $G_{3}$ and $G_{5}$, using $\delta_{G 4 X}=\delta_{G 4 X X}=0$, the scalar propagation speed squared is $c_{s}^{2}=1 /\left(1+2 \lambda_{P X}\right)$. Hence, as in the case of k-inflation, we require $\lambda_{P X} \gg 1$ to realize large non-Gaussianities. For the theories in which $P$ does not have non-linear terms in $X$ we have $c_{s}^{2}=1$ and

$$
f_{\mathrm{NL}}^{\mathrm{equil}}=\mathcal{O}\left(\epsilon_{s}, \eta_{s}\right)
$$

For example, Brans-Dicke theories described by the action $P=\omega_{\mathrm{BD}} M_{\mathrm{pl}} X / \phi-V(\phi)$ [48] belong to this class. Hence the non-Gaussianities in those theories are small (including $f(R)$ gravity where the Brans-Dicke parameter $\omega_{\mathrm{BD}}$ is 0 [61]).

The models with field derivative couplings recently studied in Refs. [49, 50] correspond to $G_{5}=F(\phi)$, in which case $\delta_{G 5 X}=0$ and $\delta_{G 5 X X}=0$. In the absence of the terms $G_{3}$ and $G_{4}$ we have

$$
c_{s}^{2}=\frac{\delta_{P X}-6 \delta_{G 5 \phi}}{\delta_{P X}\left(1+2 \lambda_{P X}\right)-6 \delta_{G 5 \phi}} .
$$

If the Lagrangian does not include non-linear terms in $X$ (like the models discussed in Ref. [49]), then $c_{s}^{2}=1$ and $f_{\mathrm{NL}}^{\text {equil }}=\mathcal{O}\left(\epsilon_{s}, \eta_{s}\right)$. This is consistent with the results found in Ref. [50].

\section{E. Potential-driven inflation with a Gauss-Bonnet term}

The action (11) even covers the Gauss-Bonnet coupling of the form $-\xi(\phi) \mathcal{G}$, where $\mathcal{G}=R^{2}-4 R_{\alpha \beta} R^{\alpha \beta}+R_{\alpha \beta \gamma \delta} R^{\alpha \beta \gamma \delta}$ is the Gauss-Bonnet term. If one chooses the following combination of $P, G_{3}, G_{4}$, and $G_{5}$

$$
\begin{array}{ll}
P=-8 \xi^{(4)}(\phi) X^{2}(3-\ln X), & G_{3}=-4 \xi^{(3)}(\phi) X(7-3 \ln X), \\
G_{4}=-4 \xi^{(2)}(\phi) X(2-\ln X), & G_{5}=4 \xi^{(1)}(\phi) \ln X,
\end{array}
$$

where $\xi^{(n)}(\phi)=\partial^{n} \xi(\phi) / \partial \phi^{n}$, one can show that the field equations following from this Lagrangian are equivalent to those derived by the Lagrangian $-\xi(\phi) \mathcal{G}[43]$.

Let us consider the case of potential-driven inflation in which the Lagrangian $X-V(\phi)$ is added to $P$ in Eq. (124). Using the choice of the functions in Eq. (124), we find that the scalar propagation speed squared is given by

$$
c_{s}^{2}=1-\frac{64 \delta_{\xi}^{2}}{\delta_{X}}\left(6 \delta_{\xi}+\delta_{X}\right),
$$


where $\delta_{X}=X /\left(M_{\mathrm{pl}}^{2} H^{2}\right)$ and $\delta_{\xi}=H \dot{\xi} / M_{\mathrm{pl}}^{2}$. This expression agrees with the one found in Ref. [38]. Since $c_{s}^{2}-1$ is a second-order quantity, the scalar propagation speed is very close to 1 . Furthermore we can show that, in this case, $\lambda / \Sigma=0$, and that the terms in Eq. (106) coming from the functions $\delta_{G 3 X}, \delta_{G 4 X X}, \delta_{G 5 X}$, and $\delta_{G 5 X X}$ are of the order



$$
f_{\mathrm{NL}}^{\text {equil }}=\frac{55}{36} \delta_{X}+\frac{5}{12} \eta_{X}+\frac{275}{81} \frac{\delta_{\xi}}{\delta_{X}}\left(4 \epsilon+2 \eta_{\xi}-\eta_{X}\right)
$$

where $\eta_{\xi}=\dot{\delta}_{\xi} /\left(H \delta_{\xi}\right)$ and $\eta_{X}=\dot{\delta}_{X} /\left(H \delta_{X}\right)$. Hence the non-linear parameter in this model is small.

\section{CONCLUSIONS}

In this paper we have evaluated the primordial non-Gaussianities generated during inflation for the models described by the action (11). Our analysis is general enough in that it covers a wide variety of single-field models having secondorder equations of motion.

The procedure to obtain the three-point correlation function of curvature perturbations is similar to that given in Ref. [45]. There are 8 shape functions which contribute to the scalar non-Gaussianities. Among them, five functions are already known to give rise to the equilateral type of non-Gaussianities. We studied the shapes of the remaining three functions and found that they can be well approximated by the same type as well. Hence the dominant contribution to the three-point correlation function comes from the equilateral configuration.

We derived the equilateral non-linear parameter $f_{\mathrm{NL}}^{\text {equil }}$ under the approximation that the slow-variation parameters defined in Eq. (10) are much smaller than 1. The formula (106) is valid for any quasi de Sitter background and thus it is convenient to apply it to concrete single-field models of inflation. In fact we used this formula to a number of models such as (A) k-inflation, (B) k-inflation with the terms $G_{i}(X)(i=3,4,5),(\mathrm{C})$ potential-driven Galileon inflation, (D) nonminimal coupling models without a non-linear term in $X,(\mathrm{E})$ potential-driven inflation with a Gauss-Bonnet term. In the models (D) and (E) we showed that $c_{s}^{2}$ is close to 1 and hence the non-Gaussianities are small. However, for the models (A), (B), (C), it is possible to realize $\left|f_{\mathrm{NL}}^{\text {equil }}\right| \gg 1$ depending on the choice of the functions $P, G_{i}(i=3,4,5)$.

The potential detectability of non-Gaussianities in future observations will allow us to distinguish between different inflationary models. In particular we expect that the precise measurement of $f_{\mathrm{NL}}^{\text {equil }}$ as well as $n_{\mathcal{R}}$ and $r$ will provide significant implications for the viability of single-field models in which $c_{s}^{2}$ is much smaller than 1 .

\section{ACKNOWLEDGEMENTS}

The work of A.D.F. and S. T. was supported by the Grant-in-Aid for Scientific Research Fund of the JSPS Nos. 10271 and 30318802. S. T. also thanks financial support for the Grant-in-Aid for Scientific Research on Innovative Areas (No. 21111006). We thank the members of the Institute for Fundamental Study for warm hospitality during our stay in Naresuan University.

Note added-While we were completing this work, we became aware of the paper by Gao and Steer [64] who calculated primordial non-Gaussianities in the same model as ours. Their formula for the bispectrum (98) is consistent with our formula (85).

\section{Appendix A: The second-order term in $f_{\mathrm{NL}}^{\text {equil (3) }}$}

The second-order term $\xi\left(\delta^{2}\right)$ in Eq. (100) is given by

$$
\begin{aligned}
\xi\left(\delta^{2}\right)= & {\left[\left(6+4 \lambda_{3 X}\right) \delta_{G 4 X}+8\left(3+\lambda_{3 X}+\lambda_{4 X}\right) \delta_{G 4 X X}+3\left(2+\lambda_{3 X}\right) \delta_{G 5 X}+\left(9+2 \lambda_{3 X}+2 \lambda_{5 X}\right) \delta_{G 5 X X}-\delta_{G 4 \phi}\left(1+\lambda_{3 X}\right)\right.} \\
& \left.-2 \delta_{G 5 \phi}\left(3+2 \lambda_{3 X}\right)\right] \delta_{G 3 X}+8 \delta_{G 4 X}^{2}+\left[16\left(5+2 \lambda_{4 X}\right) \delta_{G 4 X X}+18 \delta_{G 5 X}+8\left(4+\lambda_{5 X}\right) \delta_{G 5 X X}-2 \delta_{G 4 \phi}\right. \\
& \left.-16 \delta_{G 5 \phi}\right] \delta_{G 4 X}+64\left(2+\lambda_{4 X}\right) \delta_{G 4 X X}^{2}+\left[24\left(3+\lambda_{4 X}\right) \delta_{G 5 X}+8\left(11+2 \lambda_{4 X}+2 \lambda_{5 X}\right) \delta_{G 5 X X}-8 \delta_{G 4 \phi}\left(2+\lambda_{4 X}\right)\right. \\
& \left.-16 \delta_{G 5 \phi}\left(5+2 \lambda_{4 X}\right)\right] \delta_{G 4 X X}+9 \delta_{G 5 X}^{2}+\left[3\left(9+2 \lambda_{5 X}\right) \delta_{G 5 X X}-3 \delta_{G 4 \phi}-18 \delta_{G 5 \phi}\right] \delta_{G 5 X}+2\left(7+2 \lambda_{5 X}\right) \delta_{G 5 X X}^{2} \\
& -\left[\left(7+2 \lambda_{5 X}\right) \delta_{G 4 \phi}+8\left(4+\lambda_{5 X}\right) \delta_{G 5 \phi}\right] \delta_{G 5 X X}+8 \delta_{G 5 \phi}^{2}+2 \delta_{G 4 \phi} \delta_{G 5 \phi} .
\end{aligned}
$$


[1] A. A. Starobinsky, Phys. Lett. B 91, 99 (1980); D. Kazanas, Astrophys. J. 241 L59 (1980); K. Sato, Mon. Not. R. Astron. Soc. 195, 467 (1981); Phys. Lett. 99B, 66 (1981); A. H. Guth, Phys. Rev. D 23, 347 (1981).

[2] E. Komatsu et al., Astrophys. J. Suppl. 192, 18 (2011).

[3] B. A. Reid et al., Mon. Not. Roy. Astron. Soc. 404, L60 (2010).

[4] [PLANCK Collaboration], arXiv:astro-ph/0604069

[5] D. H. Lyth and A. Riotto, Phys. Rept. 314, 1-146 (1999).

[6] D. S. Salopek and J. R. Bond, Phys. Rev. D 42, 3936 (1990).

[7] A. Gangui, F. Lucchin, S. Matarrese and S. Mollerach, Astrophys. J. 430, 447 (1994).

[8] L. Verde, L. M. Wang, A. Heavens and M. Kamionkowski, Mon. Not. Roy. Astron. Soc. 313, L141 (2000).

[9] E. Komatsu and D. N. Spergel, Phys. Rev. D63, 063002 (2001).

[10] V. Acquaviva, N. Bartolo, S. Matarrese and A. Riotto, Nucl. Phys. B667, 119-148 (2003).

[11] J. M. Maldacena, JHEP 0305, 013 (2003).

[12] G. I. Rigopoulos and E. P. S. Shellard, Phys. Rev. D 68, 123518 (2003); JCAP 0510, 006 (2005).

[13] D. H. Lyth and Y. Rodriguez, Phys. Rev. Lett. 95, 121302 (2005); D. H. Lyth and Y. Rodriguez, Phys. Rev. D 71, 123508 (2005); D. H. Lyth, K. A. Malik and M. Sasaki, JCAP 0505, 004 (2005); C. T. Byrnes, M. Sasaki and D. Wands, Phys. Rev. D 74, 123519 (2006).

[14] P. Creminelli, A. Nicolis, L. Senatore, M. Tegmark and M. Zaldarriaga, JCAP 0605, 004 (2006).

[15] C. Armendariz-Picon, T. Damour and V. F. Mukhanov, Phys. Lett. B 458, 209 (1999).

[16] E. Silverstein and D. Tong, Phys. Rev. D 70, 103505 (2004).

[17] M. Alishahiha, E. Silverstein and D. Tong, Phys. Rev. D 70, 123505 (2004).

[18] N. Arkani-Hamed, H. C. Cheng, M. A. Luty and S. Mukohyama, JHEP 0405, 074 (2004); N. Arkani-Hamed, P. Creminelli, S. Mukohyama and M. Zaldarriaga, JCAP 0404, 001 (2004).

[19] F. Piazza and S. Tsujikawa, JCAP 0407, 004 (2004).

[20] T. Kobayashi, M. Yamaguchi and J. Yokoyama, Phys. Rev. Lett. 105, 231302 (2010).

[21] C. Burrage, C. de Rham, D. Seery and A. J. Tolley, JCAP 1101, 014 (2011).

[22] D. Seery and J. E. Lidsey, JCAP 0506, 003 (2005).

[23] A. Gruzinov, Phys. Rev. D 71, 027301 (2005).

[24] X. Chen, M. x. Huang, S. Kachru and G. Shiu, JCAP 0701, 002 (2007); X. Chen, R. Easther and E. A. Lim, JCAP 0706, 023 (2007); JCAP 0804, 010 (2008).

[25] J. Ohashi and S. Tsujikawa, Phys. Rev. D83, 103522 (2011).

[26] M. Ostrogradski, Mem. Ac. St. Petersbourg VI 4, 385 (1850).

[27] G. W. Horndeski, Int. J. Theor. Phys. 10, 363-384 (1974).

[28] G. R. Dvali, G. Gabadadze and M. Porrati, Phys. Lett. B 485, 208 (2000).

[29] A. Nicolis, R. Rattazzi and E. Trincherini, Phys. Rev. D 79, 064036 (2009).

[30] C. Deffayet, G. Esposito-Farese and A. Vikman, Phys. Rev. D79, 084003 (2009); C. Deffayet, S. Deser, G. Esposito-Farese, Phys. Rev. D80, 064015 (2009).

[31] C. de Rham and A. J. Tolley, JCAP 1005, 015 (2010); C. Burrage, C. de Rham and L. Heisenberg, JCAP 1105,025 (2011); C. de Rham and L. Heisenberg, arXiv:1106.3312 [hep-th].

[32] P. Creminelli, A. Nicolis and E. Trincherini, JCAP 1011, 021 (2010); P. Creminelli, G. D'Amico, M. Musso, J. Norena and E. Trincherini, JCAP 1102, 006 (2011).

[33] K. Kamada, T. Kobayashi, M. Yamaguchi and J. Yokoyama, Phys. Rev. D83, 083515 (2011).

[34] A. Naruko and M. Sasaki, Class. Quant. Grav. 28, 072001 (2011).

[35] K. Van Acoleyen and J. Van Doorsselaere, Phys. Rev. D83, 084025 (2011).

[36] G. Goon, K. Hinterbichler, M. Trodden, arXiv:1103.5745 [hep-th]]; G. Goon, K. Hinterbichler, M. Trodden, arXiv:1103.6029 [hep-th].

[37] S. Renaux-Petel, arXiv:1105.6366 [astro-ph.CO].

[38] A. De Felice, S. Tsujikawa, J. Elliston and R. Tavakol, arXiv:1105.4685 [astro-ph.CO].

[39] X. Gao, arXiv:1106.0292 [astro-ph.CO].

[40] N. Chow and J. Khoury, Phys. Rev. D 80, 024037 (2009); F. P. Silva and K. Koyama, Phys. Rev. D 80, 121301 (2009); T. Kobayashi, H. Tashiro and D. Suzuki, Phys. Rev. D 81, 063513 (2010); T. Kobayashi, Phys. Rev. D 81, 103533 (2010); R. Gannouji and M. Sami, Phys. Rev. D 82, 024011 (2010); A. De Felice and S. Tsujikawa, JCAP 1007, 024 (2010); A. De Felice, S. Mukohyama and S. Tsujikawa, Phys. Rev. D 82, 023524 (2010); C. Deffayet, O. Pujolas, I. Sawicki and A. Vikman, JCAP 1010, 026 (2010); A. De Felice and S. Tsujikawa, Phys. Rev. Lett. 105, 111301 (2010); arXiv:1008.4236 [hep-th]; S. Nesseris, A. De Felice and S. Tsujikawa, Phys. Rev. D 82, 124054 (2010); D. F. Mota, M. Sandstad and T. Zlosnik, JHEP 1012, 051 (2010); R. Kimura and K. Yamamoto, JCAP 1104, 025 (2011); A. De Felice, R. Kase and S. Tsujikawa, Phys. Rev. D 83, 043515 (2011); O. Pujolas, I. Sawicki and A. Vikman, arXiv:1103.5360 [hep-th]; A. De Felice, T. Kobayashi and S. Tsujikawa, arXiv:1108.4242 [gr-qc].

[41] C. Deffayet, X. Gao, D. A. Steer and G. Zahariade, arXiv:1103.3260 [hep-th].

[42] C. Charmousis, E. J. Copeland, A. Padilla and P. M. Saffin, arXiv:1106.2000 [hep-th].

[43] T. Kobayashi, M. Yamaguchi and J. Yokoyama, arXiv:1105.5723 [hep-th].

[44] S. Mizuno and K. Koyama, Phys. Rev. D 82, 103518 (2010). 
[45] A. De Felice and S. Tsujikawa, JCAP 1104, 029 (2011).

[46] T. Kobayashi, M. Yamaguchi and J. Yokoyama, Phys. Rev. D 83, 103524 (2011).

[47] T. Qiu and K. C. Yang, arXiv:1012.1697 [hep-th].

[48] C. Brans and R. H. Dicke, Phys. Rev. 124, 925-935 (1961); Y. Fujii and K. Maeda, "The scalar-tensor theory of gravitation," Cambridge, USA: Univ. Pr., 240 p (2003).

[49] C. Germani and A. Kehagias, Phys. Rev. Lett. 105, 011302 (2010); Phys. Rev. Lett. 106, 161302 (2011).

[50] C. Germani and Y. Watanabe, arXiv:1106.0502 [astro-ph.CO].

[51] T. P. Sotiriou and V. Faraoni, Rev. Mod. Phys. 82, 451 (2010); A. De Felice and S. Tsujikawa, Living Rev. Rel. 13, 3 (2010).

[52] R. R. Metsaev and A. A. Tseytlin, Nucl. Phys. B 293, 385 (1987); I. Antoniadis, J. Rizos and K. Tamvakis, Nucl. Phys. B 415, 497 (1994); M. Gasperini, M. Maggiore and G. Veneziano, Nucl. Phys. B 494, 315 (1997); R. Brustein and R. Madden, Phys. Rev. D 57, 712 (1998); C. Cartier, E. J. Copeland and R. Madden, JHEP 0001, 035 (2000); Z. K. Guo, N. Ohta and S. Tsujikawa, Phys. Rev. D75, 023520 (2007).

[53] R. L. Arnowitt, S. Deser and C. W. Misner, arXiv:gr-qc/0405109

[54] J. M. Bardeen, Phys. Rev. D 22, 1882 (1980); H. Kodama and M. Sasaki, Prog. Theor. Phys. Suppl. 78, 1 (1984); V. F. Mukhanov, H. A. Feldman and R. H. Brandenberger, Phys. Rept. 215, 203 (1992); B. A. Bassett, S. Tsujikawa and D. Wands, Rev. Mod. Phys. 78, 537 (2006); K. A. Malik and D. Wands, Phys. Rept. 475, 1-51 (2009).

[55] H. Collins, arXiv:1101.1308 [astro-ph.CO].

[56] F. Arroja and T. Tanaka, JCAP 1105, 005 (2011); G. Rigopoulos, Phys. Rev. D84, 021301 (2011).

[57] K. Koyama, Class. Quant. Grav. 27, 124001 (2010).

[58] Y. i. Takamizu, S. Mukohyama, M. Sasaki and Y. Tanaka, JCAP 1006, 019 (2010).

[59] D. Babich, P. Creminelli and M. Zaldarriaga, JCAP 0408, 009 (2004).

[60] J. R. Fergusson and E. P. S. Shellard, Phys. Rev. D 80, 043510 (2009).

[61] J. O'Hanlon, Phys. Rev. Lett. 29, 137 (1972); T. Chiba, Phys. Lett. B 575, 1 (2003).

[62] J. Garriga and V. F. Mukhanov, Phys. Lett. B 458, 219 (1999).

[63] J. -c. Hwang, H. Noh, Phys. Rev. D66, 084009 (2002).

[64] X. Gao and D. A. Steer, arXiv:1107.2642 [astro-ph.CO]. 